\title{
NANOFERROICS: NEW EFFECTS, PROPERTIES, POSSIBILITIES
}

\author{
V. Kapustianyk \\ Scientific-Technical and Educational Center of Low Temperature Studies, \\ Research and Educational Center "FRACTAL", \\ Department for Solid State Physics, Ivan Franko National University of Lviv \\ 50, Drahomanov St., UA-79005, Ukraine \\ e-mail: kapustianyk@yahoo.co.uk
}

(Received January 25, 2013)

\begin{abstract}
The latest results concerning the quantum confinement and other size effects in the nanoferroics are reviewed. The main attention was devoted to the spectral, dielectric and nonlinear optics properties of the nanocomposites based on the single crystals $\left(\mathrm{NH}_{2}\left(\mathrm{C}_{2} \mathrm{H}_{5}\right)_{2}\right)_{2} \mathrm{CuCl}_{4}$ (furthermore DEACC) and $\left(\mathrm{N}\left(\mathrm{C}_{2} \mathrm{H}_{5}\right)_{4}\right)_{2} \mathrm{CoCl}_{2} \mathrm{Br}_{2}$ (TEACCB). The peculiarities of the magnetic and dielectric properties were discussed on the example of the low dimensional ferroic $\left(\mathrm{C}_{2} \mathrm{H}_{5} \mathrm{NH}_{3}\right)_{2} \mathrm{CuCl}_{4}$ (EACC). The physical nature of the principally new effects and properties of the nanostructured ferroics is considered. It has been shown that due to the embedding into a polymer matrix in the NC state the ferroic becomes very attractive from the technological point of view. The hygroscopic, brittle and thermally unstable crystals grown from aqueous solution are transformed in such a way that they would be used as principally new nonlinear optics elements, piezotransducers, sensitive materials for sensors, etc.
\end{abstract}

Key words: nanoferroic, nanocomposite, quantum confinement, multiferroic, thermochromic effect, dielectric dispersion, nonlinear optics, phase transitions.

PACS number(s): 72.22.-d, 77.22.Ch, 77.22Gm, 89.20.Sv, 75.85.+t, 81.05.Lg, 75.50.Dd, 75.50.Ee

\section{INTRODUCTION}

The ferroelectric, ferroeleastic, ferromagnetic and related materials well known to the scientists working in the field of solid states physics would be considered as the first order ferroics. In some materials related to the class of the higher order ferroics one can observe co-existence of two or more order parameters. Nature and people created a large variety of these materials able to react very similarly on the influence of the external fields. The ferroics ineract with the external electromagnetic, elastic and temperature fields manifesting four important collective effects connected with the change of the free energy, namely: phase transition, followed by the change of the lattice symmetry; anomalous temperature behaviour of the susceptibilities, elastic constants, heat capacitance and thermal expansion coefficients; transformation of the domain structure; nonlinear hysteresis change of the parameters. The largest response of such materials usually is found to be in the vicinity of the phase transitions, where the domain structure is destabilized due to the transformation of the lattice. The more symmetric phase in this case becomes stable [1].

The properties of single crystalline ferroics are discussed in the numerous reviews. The data base concerning the ferroics and structural changes at their phase transitions one can find in [2]. The single crystalline ferroics with organic cations were considered in details in the author's publications [3,4]. The experimental studies of the structurally incommensurate crystal phases in ferroics are described in [5]. A considerable large inter- est in ferroics of different types is connected with their applications in different branches of human activities. In many cases the polycrystalline samples, first of all ceramic, were found to be much more attractive from the point of view of technology.

For a long time the single crystalline ferroics with an organic cation, usually grown from the aqueous solution, were not considered as attractive materials for engineering and electronics. In this review on the example of a few initial materials of this family we are going to demonstrate that the creation of the nanocomposites on their basis could open the new possibilities of practical applications in all respects. The main attention would be devoted to the nanocomposites on the basis of the single crystals $\left(\mathrm{NH}_{2}\left(\mathrm{C}_{2} \mathrm{H}_{5}\right)_{2}\right)_{2} \mathrm{CuCl}_{4}$ (furthermore DEACC) and $\left(\mathrm{N}\left(\mathrm{C}_{2} \mathrm{H}_{5}\right)_{4}\right)_{2} \mathrm{CoCl}_{2} \mathrm{Br}_{2}(\mathrm{TEACCB})$ and to the low dimensional ferroic $\left(\mathrm{C}_{2} \mathrm{H}_{5} \mathrm{NH}_{3}\right)_{2} \mathrm{CuCl}_{4}$ (EACC).

Numerous crystals containing alkylammonium cations and inorganic anions are under detailed investigation of a great number of scientists due to the complicated sequences of different type phase transitions (PTs), including transitions into the incommensurate phase, connected with the dynamics of the organic cations and inorganic anions. Unfortunately, their technical application is restrained because of high hygroscopicity, brittleness and damaging caused by overheating above $100^{\circ} \mathrm{C}$. It was shown [6-9] that the incorporation of nanocrystals (NC) into a polymer matrix may open a new opportunity for the application of the semiconductor, oxide and dielectric NC as materials for photo-operated nonlinear optics, photorefractive devices as well as for optically operat- 


\section{KAPUSTIANYK}

ed modulators, $Q$-switches, deflectors etc. Recently the tetraethylammonium tetrahalogenometallic compounds $\left[\mathrm{N}\left(\mathrm{C}_{2} \mathrm{H}_{5}\right)_{4}\right]_{2} \mathrm{MeX}_{4}$ (abbreviately TEAMeX; $M e=\mathrm{Co}$, $\mathrm{Zn}, \mathrm{Cu}, \mathrm{Mn} ; X=\mathrm{Cl}, \mathrm{Br})$ as well as TEACCB have been the subject of considerable attention [8,10-13]. According to [8] the bulk TEACCB crystal undergoes two structural phase transitions below room temperature:

$$
\text { Improper ferroelectric } \leftarrow \underset{T_{2}, 232^{\circ} \mathrm{K} \text { (at heating) }}{T_{2}, 224^{\circ} \mathrm{K} \text { (on cooling) }} \leftarrow T_{1}, 249^{\circ} \mathrm{K} \leftarrow \mathrm{P} 42 / \mathrm{nmc}
$$

These crystals are of particular interest because they combine organic cations with the inorganic anions. In this case the tetraethyl cations with the prevailingly covalence type of bonds coexist with the prevailingly ionic anionic tetrahedra. Moreover, due to the availability of the transition metal complexes one can expect a substantial influence of the $p-d$ charge transfer playing an important role in the optical properties as well as in the electron-phonon interactions. Diethylammonium copper chloride (DEACC) crystals grown from an aqueous solution possess a clear discontinuous thermochromic phase transition at $311 \mathrm{~K}$ (at heating) accompanied by a sharp change in sample colour from deep green to pale yellow caused by the change of the copper ion coordination. Such transitions are closely related to the principal spectral bands corresponding to the intra-center $d-d$ transition of $\mathrm{Cu}^{2+}$ ion [14]. The crystal structure of DEACC consists of $\mathrm{DEA}^{+}$cations and metalhalogen complexes (MHCs) chemically bonded by hydrogen bonds. The high-temperature phase possesses a distorted tetrahedral environment of the copper ion, whereas in the low-temperature phase, the tetrahedral and planar square coordinations coexist $[14,15]$. At room temperature the crystal belongs to the space group $\mathrm{P} 21 / n$ as a low temperature phase. The lattice parameters of the unit cell are $a=7.362 \AA, b=15.025 \AA, c=45.193 \AA$, $\beta=89.94^{\circ}$ and $z=12$. One asymmetrical structural group includes three tetrahedrally and nine octahedrally coordinated $\left[\mathrm{CuCl}_{4}\right]^{2-}$ ions in an exceptionally large cell. In the first approximation, they can be considered to possess the $\mathrm{D}_{2 d}$ symmetry. The crystal structure in the high-temperature phase has the space group $\mathrm{P} 21 / c$ $\left(a=25.055 \AA, b=10.531 \AA, c=15.455 \AA, \beta=100.6^{\circ}\right.$ and $z=8$ ) [15]. In this phase, two independent crystallographic $\left[\mathrm{CuCl}_{4}\right]^{2-}$ ions have the shape of a distorted tetrahedron. Due to their specific thermochromic properties DEACC crystals are considered to be promising materials for sensors, in particular, for thermography and for optical data storage. They can be very interesting from the point of view of photoinduced and nonlinear optical properties for the corresponding nanocomposites (nanocrystals) incorporated into the polymer matrices [16].

Vigorous research efforts in magnetoelectric (ME) effects in recent years [17-19] have stimulated both exploration of magnetoelectric materials [20] and investigation of previously known ferroic compounds.



Fig. 1. (Color online) Schematic view of the orthorhombic crystal structure for EACC (300 K), the Jahn-Teller distorted copper chloride octahedra are highlighted. The ammonium heads of the organic cations form strong hydrogen bonds to any of the eight halides that results in the reorientational disorder of the bonding scheme; for simplicity the hydrogens of the groups are not shown. The arrows at the copper sites are a graphical representation of the spin configuration involving adjacent layers at lower temperatures [21].

In that respect revisiting previously known hybrid crystal structures comprising metal ions in complex with organic molecules brings-up an attractive opportunity to explore their magnetoelectric properties that are highly promising for information storage [22] or electric field controlled magnetic sensors. A good candidate to meet this challenge is the family of compounds with the com- 
position $\left(\mathrm{C}_{n} \mathrm{H}_{2 n+1} \mathrm{NH}_{3}\right)_{2} M \mathrm{Cl}_{4}$, where $M$ is a divalent metal $\left(M=\mathrm{Mn}^{2+}, \mathrm{Cd}^{2+}, \mathrm{Fe}^{2+}\right.$ and $\left.\mathrm{Cu}^{2+}\right)$. This series crystallizes in the layered perovskite structure [23-26], consisting of infinite, staggered layers of corner-sharing $M \mathrm{Cl}_{6}$ octahedra interleaved by alkylammonium cations $\left[\mathrm{C}_{n} \mathrm{H}_{2 n+1} \mathrm{NH}_{3}^{+}\right.$; abbreviated as MA for methylammonium $(n=1)$, EA for ethylammonium $(n=2)$, etc]. The cavities between octahedra are occupied by the ammonium heads of the organic cations, which importantly form strong $\mathrm{N}-\mathrm{H} \ldots \mathrm{Cl}$ hydrogen bonds to any of the eight halides. In this structure, adjacent layers are stacked upon one another through van der Waals force between terminal alkyl groups. It is worth noting that the choice of the hydrogen bonding scheme is important for determining the orientation and conformation of the organic molecules within the layered hybrid structure and in effect can influence the temperature evolution of the structural phase transitions in the perovskite structure [27-29].

The system of interest, the $\left(\mathrm{C}_{2} \mathrm{H}_{5} \mathrm{NH}_{3}\right)_{2} \mathrm{CuCl}_{4}$ (EACC) compound crystallizes with an orthorhombic cell (space group, Pbca; $a=21.18 \AA, b=7.47 \AA, c=$ $7.35 \AA$ ) [19] at room-temperature. It comprises Jahneller $(\mathrm{J}-\mathrm{T})$ distorted $\mathrm{Cu}^{2+}$ layers separated by two layers of ethylammonium groups (Fig. 1). Notably, this compound undergoes a series of structural phase transitions [30] as a consequence of modifications not only due to the $\mathrm{J}-\mathrm{T}$ effect, but importantly due to the arrangement (orientational order and conformation of the organic molecules) of the ethylammonium chains $[31,32]$. With the temperature increase these entail: Triclinic $\left(T_{4}=232 \mathrm{~K}\right) \rightarrow$ Pbca $\left(T_{3}=330 \mathrm{~K}\right) \rightarrow$ Rhombic $\left(T_{2}=356 \mathrm{~K}\right) \rightarrow \mathrm{P} 21 / c\left(T_{1}=364 \mathrm{~K}\right) \rightarrow$ Bbcm.

\section{TECHNOLOGY AND EXPERIMENTAL DETAILS}

The single crystals of ferroics considered in this review were grown from aqueous solutions of the corresponding salts taken in the stoichiometric ratio. The preparation of the nanocomposite consists of the following steps. The synthesized single crystals were crushed using external acoustical focused field to the sizes within the range of 8-100 $\mathrm{nm}$ with dispersion of the sizes varied between the $3-14 \mathrm{~nm}$ depending on the purpose of investigations. Samples of the certain sizes were picked out using a membrane filter. The powder-like NC specimens were dispersed in the liquid oligoetheracrylate (OEA) photopolymer composition. The solidification process and electropoling homogenization were performed by the method described in [6]. Nitrogen laser with $\lambda=377 \mathrm{~nm}$ and the photon energy power density about $65 \mathrm{~W} / \mathrm{cm}^{2}$ was used for the solidification. In other cases the $\mathrm{NC}$ were incorporated into the polymethylmethacrylate (PMMA) matrix.

a)

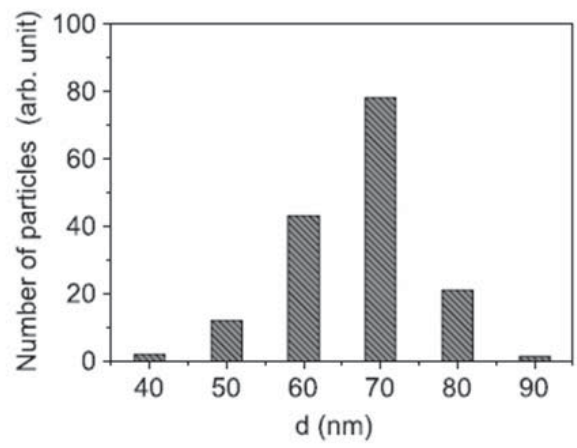

c)
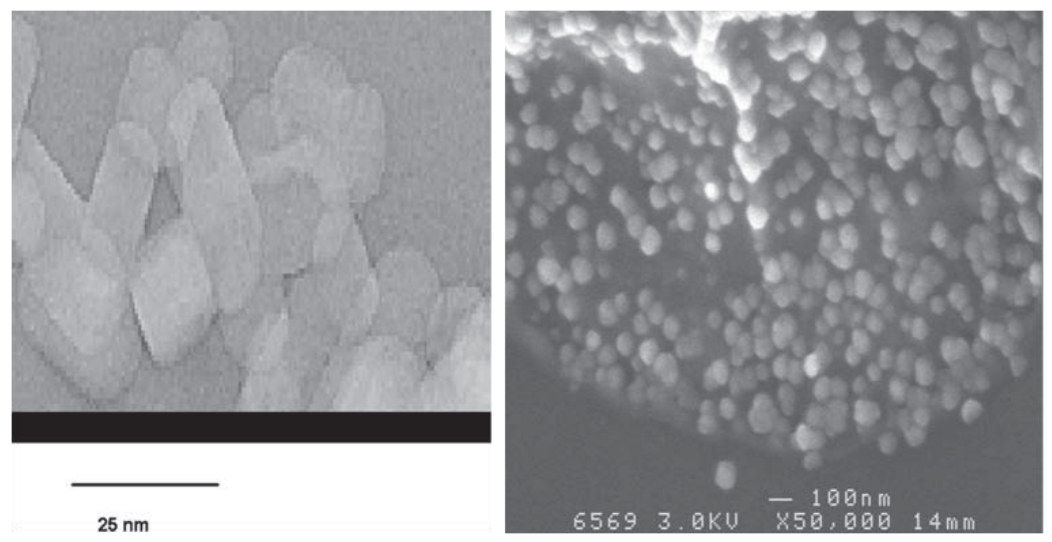

Fig. 2. Size distribution (a) and typical TEM (b) and SEM (c) image of the ferroic nanocrystals in the studied polymer composites. 


\section{KAPUSTIANYK}

In this case the prepared nanocrystals were first mixed with polymethylmethacrylate in the appropriate ratio and then dissolved in the organic solvent. After evaporating the solvent the samples with different thickness and concentration of the nanocrystals were obtained.

In many cases it is important to determine the size dispersion of the nanoparticles. Usually it is measured by the nanoparticle size analyzer such as Brookhaven 90 . Figure 2a presents an example of such a distribution in the TEACCB/PMMA composite. One can see that most of the nanocrystals have sizes in the vicinity of $70 \mathrm{~nm}$ with some asymmetry of size dispersion.

The size distribution and uniformity of the nanocrystals in the polymer matrix usually are tested by SEM and TEM methods. A typical micrographs of the nanocomposite based on DEACC crystals are presented in Fig. 2 $\mathrm{b}, \mathrm{c}$, which indicates the incorporation of the nanocrystals into the polymer matrix and formation of the nanocomposite. The obtained size distribution of nanocrystals in the prepared samples would correlate with those obtained with nanoparticle size analyzer. The X-ray diffraction data of the powder sample were collected using an HZG-4A powder diffractometer $(\mathrm{Cu} \mathrm{Ka}$ radiation, $10^{\circ} \leq 2 \theta \leq 7^{\circ}$ and step scan mode with a step size of $0.02^{\circ}$ ). The crystal structure refinements were determined using the CSD program package [33]. The experimental details concerning the measurements of the dielectric dispersion and magnetic properties are described in $[9,34]$. The polarized and nonpolarized absorption spectra of the bulk crystals and nanocomposites were investigated within the spectral range $250-950 \mathrm{~nm}$ by the automated site based on a ZMR-3 monochromator with a spectral resolution of $1 \mathrm{~nm}$. DSC measurements were done with the commercial Netzsch Simultaneous Thermal Analyzer STA409C [35]. The scheme of the nonlinear optical set-up as well as other experimental details concerning the second and third harmonic generation would be found in [36].

\section{RESONANCE DIELECTRIC DISPERSION OF THE FERROIC NANOCRYSTALS INCORPORATED INTO THE POLYMER MATRIX}

In this part we demonstrate how the dielectric properties of TEACCB solid solution within the frequency range of $3 \cdot 10^{5}-2.6 \cdot 10^{9} \mathrm{~Hz}$ can be dramatically changed when the nanoparticles of this crystal are dispersed within the PMMA polymer matrix.

\section{A. Experimental results}

The dielectric properties of the pure PMMA were investigated fairly well $[37,38]$. No significant frequency dispersion was detected by us within the $3 \cdot 10^{5}-$ $2.6 \cdot 10^{9} \mathrm{~Hz}$ frequency range neither for pure PMMA nor for the bulk TEACCB crystal (see Fig. 3). Both compounds demonstrate the flat frequency dependence of both the real and imaginary parts of $\varepsilon$. However, the frequency dispersion of the dielectric permittivity of the combined TEACCB NC-PMMA matrix material differs drastically from those obtained for pure compounds. As is seen from Fig. 4, there is a clearly pronounced resonance frequency dispersion of both the real and imaginary parts of the dielectric permittivity. We tested the composite compounds with three different concentrations of nanocrystals $(3,5$ and $7 \%)$. The resonance frequency was found to be near $1.3 \mathrm{GHz}$ and does not depend on $\mathrm{NC}$ concentration. However, the increase of the TEA-CCB NC concentration results in an increase of the resonance values of both $\varepsilon^{\prime}$ and $\varepsilon^{\prime \prime}$. For the highest $\mathrm{NC}$ concentration $(7 \%)$ the real part of the permittivity demonstrates negative values within a certain frequency range above the resonance.
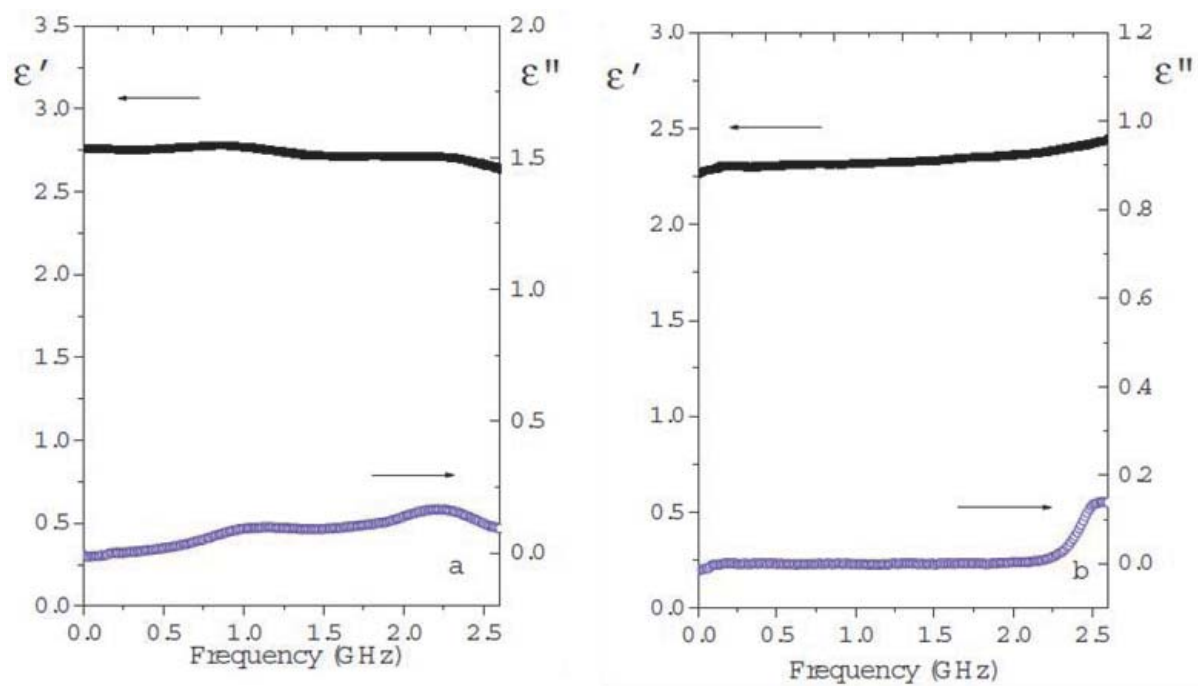

Fig. 3. Frequency dependences of the real $\varepsilon^{\prime}$ and imaginary $\varepsilon^{\prime \prime}$ parts of the dielectric permittivity for pure PMMA (a) and for bulk TEACCB (b) obtained at $T=293 \mathrm{~K}$. 

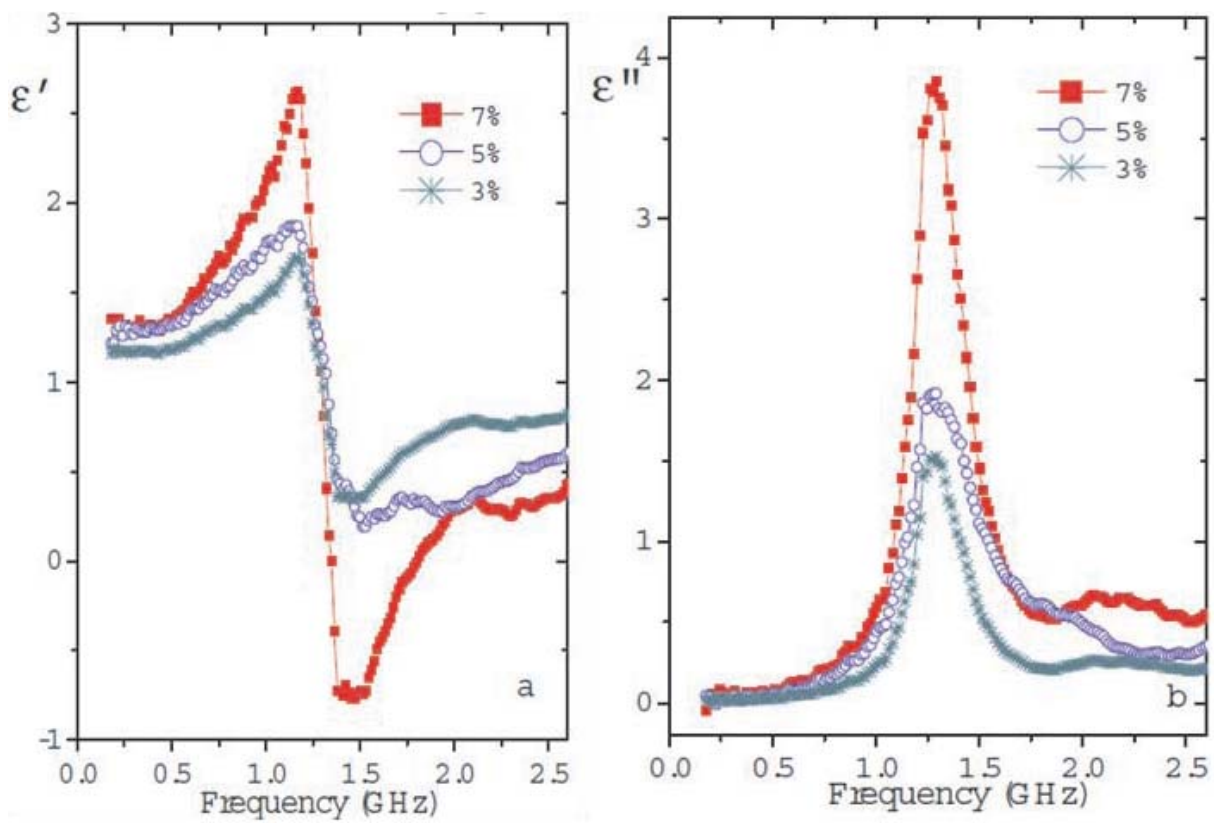

Fig. 4. The real (a) and imaginary (b) parts of the dielectric permittivity as a function of frequency for PMMA+TEACCB with NCs.

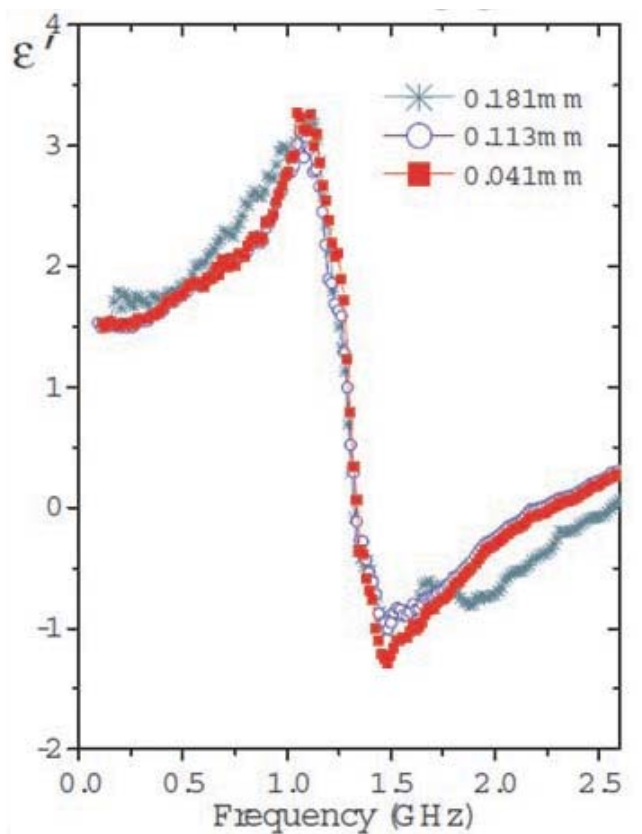

Fig. 5. Frequency dispersion of the real part of the dielectric permittivity for PMMA+TEACCB samples at various thicknesses (at room temperature).

It is worth noting that the dielectric resonance observed by us has no relation to the dimensional resonance effect connected with a macroscopic sample thickness [39]. In order to prove this we have performed the experiment with samples of different thicknesses. The obtained results (Fig. 5) show that the sample thickness at constant $\mathrm{NC}$ concentration does not influence either resonance frequency or resonance value of $\varepsilon$. Therefore one can conclude that this phenomenon is connected with the substantial physical properties of the material and not with its geometry.

In order to gain more information about the physical mechanism of this phenomenon, investigations of the dielectric permittivity were carried out for various temperatures and the results are plotted in Fig. 6 (for legibility the number of temperature dependencies was limited to three temperature points). As one can see from this figure the resonance frequency decreases upon cooling. This is accompanied by the diminution of the resonance values of both $\varepsilon^{\prime}$ and $\varepsilon^{\prime \prime}$.

In order to test the presence of the structural acentricity in both the TEACCB compound and PMMA+TEACCB composite we have carried out a Xray powder diffraction and SHG investigation, respectively. X-ray powder diffraction studies have revealed that the TEACCB is isomorphous with TEA-Co $X_{4}(X=$ $\mathrm{Cl}, \mathrm{Br}$ ) compounds, which belong to the P42/nmc group of symmetry [40-42]. Experimental X-ray diffraction patterns of TEACCB powder and that simulated using the CSD program for $\mathrm{P} 42 / \mathrm{nmc}$ symmetry are shown in Fig. 7. The unit cell parameters were found to be $a=b=9.028(1) \AA, c=15.404(2) \AA$. They are very close to those in TEACo $X_{4}$ crystals [40-42]. Therefore, one may infer that the centric P42/nmc symmetry is relevant for the TEACCB crystal at room temperature.

The investigations of the second harmonic generation (SHG) of composite material were performed at room temperature. We have found that the maximally achieved value of the effective second-order susceptibility was equal to about $0.56 \mathrm{pm} \mathrm{V}^{-1}$. This value is certainly lower compared to the inorganic crystals like BBO (about $4.2 \mathrm{pm} \mathrm{V}^{-1}$ ). However, it may be very critical that the reliable SHG signal exists in the investigated composites reflecting the existence of local acentricity in the investigated materials. 


\section{KAPUSTIANYK}
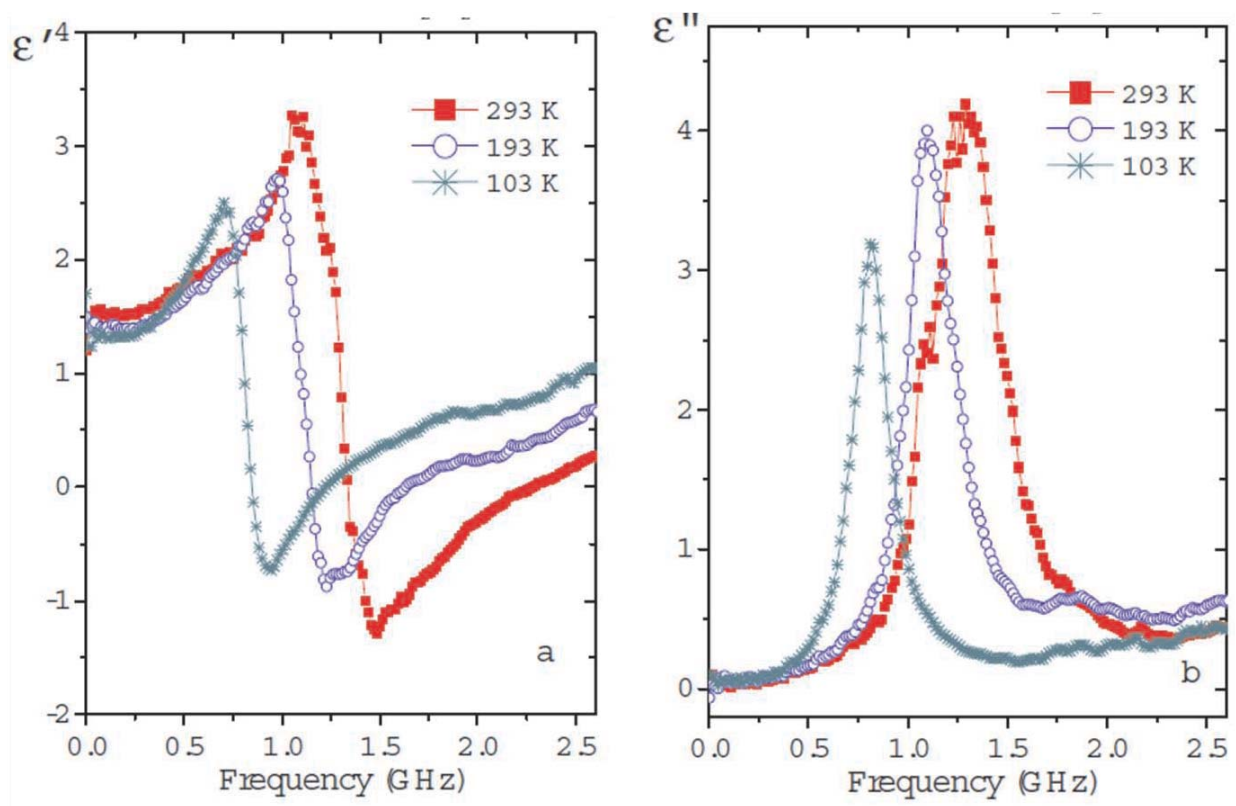

Fig. 6. Frequency dependences of the real (a) and imaginary (b) parts of the dielectric permittivity for PMMA + TEACCB $(7 \%)$ composite taken at various temperatures.

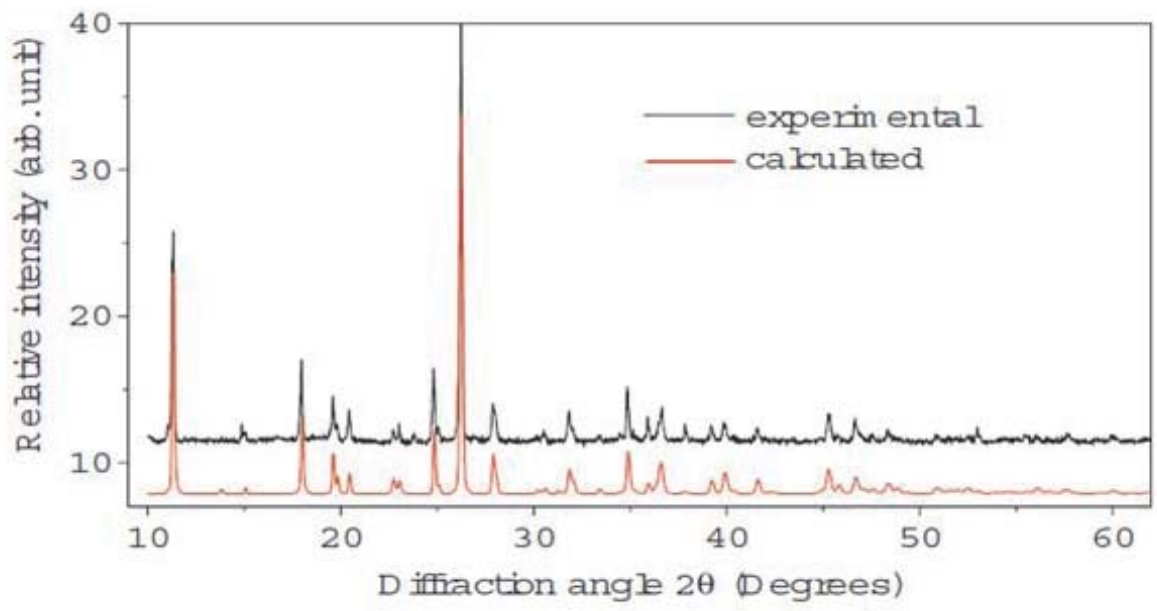

Fig. 7. X-ray powder diffraction patterns of the TEACCB crystal measured (upper line) at room temperature and calculated (lower line) using the CSD program.

\section{B. Discussion}

The appearance of negative dielectric permittivity is not an unusual phenomenon in solid state physics. The following relation [43]:

$$
\varepsilon=\varepsilon_{\infty} \prod \frac{\omega^{2}-\omega_{j \mathrm{LO}}^{2}-i \omega \gamma_{\alpha}}{\omega^{2}-\omega_{j \mathrm{TO}}^{2}-i \omega \gamma_{\alpha}}
$$

is generally used for the description of IR spectra in dielectrics. The dielectric permittivity manifests the negative value within the $\omega_{\mathrm{TO}}<\omega<\omega_{\mathrm{LO}}$ frequency region, where $\omega_{\mathrm{TO}}$ and $\omega_{\mathrm{LO}}$ are the transverse and longitudinal phonon mode frequencies, respectively. However, the typical frequency range where this phenomenon occurs lies in the far- and mid-infrared region $\left(10^{12}-10^{14} \mathrm{~Hz}\right)$. We believe that the reason for the observed dielectric anomaly may originate from the electromechanical piezoelectric resonance of TEACCB NCs. However, as follows from our X-ray data, the bulk TEACCB crystal manifests the centric P42/nmc symmetry at room temperature and, hence, would not have piezoelectricity. On the other hand, composite PMMA+ TEACCB material demonstrates the macroscopic acentricity which was clearly observed in our SHG experiment. It is obvious that the total acentricity of the composite compound may only be evoked by the acentric symmetry of TEACCB NC. Apparently, the PMMA matrix should reveal the electret properties [44] polarizing the NCs dispersed in it. The influence of the internal electret field of PMMA should play the key role in creating the acentrici- 
ty in the TEACCB NCs as well as in the temperature shift of the resonance frequency. Some analogy could be made with the poling of piezoceramics. The resonance frequency depends on the value of the applied poling electric field [44]. Moreover, the additional investigation revealed the presence of the pyroelectric polarization in PMMA+TEACCB composites (corresponding results will be presented elsewhere). It was shown $[45,46]$ that piezoelectric resonant spectra can be described by the model of multiple forced-dumped oscillator:

$$
\begin{aligned}
\varepsilon^{*}(\omega) & =\varepsilon^{\prime}(\omega)+i \varepsilon^{\prime \prime}(\omega) \\
& =\varepsilon_{c d}+\sum_{k} A_{k} \frac{\left(\omega^{2}-\omega_{0 k}^{2}\right)-i 2 \gamma_{k} \omega}{\left(\omega^{2}-\omega_{0 k}^{2}\right)+4 \gamma_{k}^{2} \omega},
\end{aligned}
$$

where $\omega_{0 k}^{2}(d)$ and $A_{k}(d)$ are the dimension-dependent resonant (or characteristic) frequency and amplitude of the i-th oscillator, respectively. $\gamma_{k}(d)$ and $\varepsilon_{c d}$ are the dumping factor and "clamped" dielectric permittivity, respectively. In this model, positive and negative ionic units oscillate in opposite directions under the influence of an applied AC electric field. Such an approach is similar to that described by equation (1), but it gives a lower frequency due to the cooperative character of the considered effect. The dimensional dependence of the parameters in equation (2) occurs since the piezoelectric resonance is a macroscopic cooperative phenomenon in which the sample (NC) size, shape and boundary conditions play essential roles. The detailed theoretical description could be found in [45-47]. According to the structural data the oscillating charges in TEACCB can be the $\left[\mathrm{CoCl}_{2} \mathrm{Br}_{2}\right]^{2-}$ anion from one side and a couple of $\left[\mathrm{N}\left(\mathrm{C}_{2} \mathrm{H}_{5}\right)_{4}\right]^{+}$cations as the second part of the oscillating unit. Such an assumption fairly well correlates with the results of the low frequency dielectric studies of TEACCB bulk crystal that revealed dispersion connected with cooperative motion of the oppositely charged complexes mentioned [48].
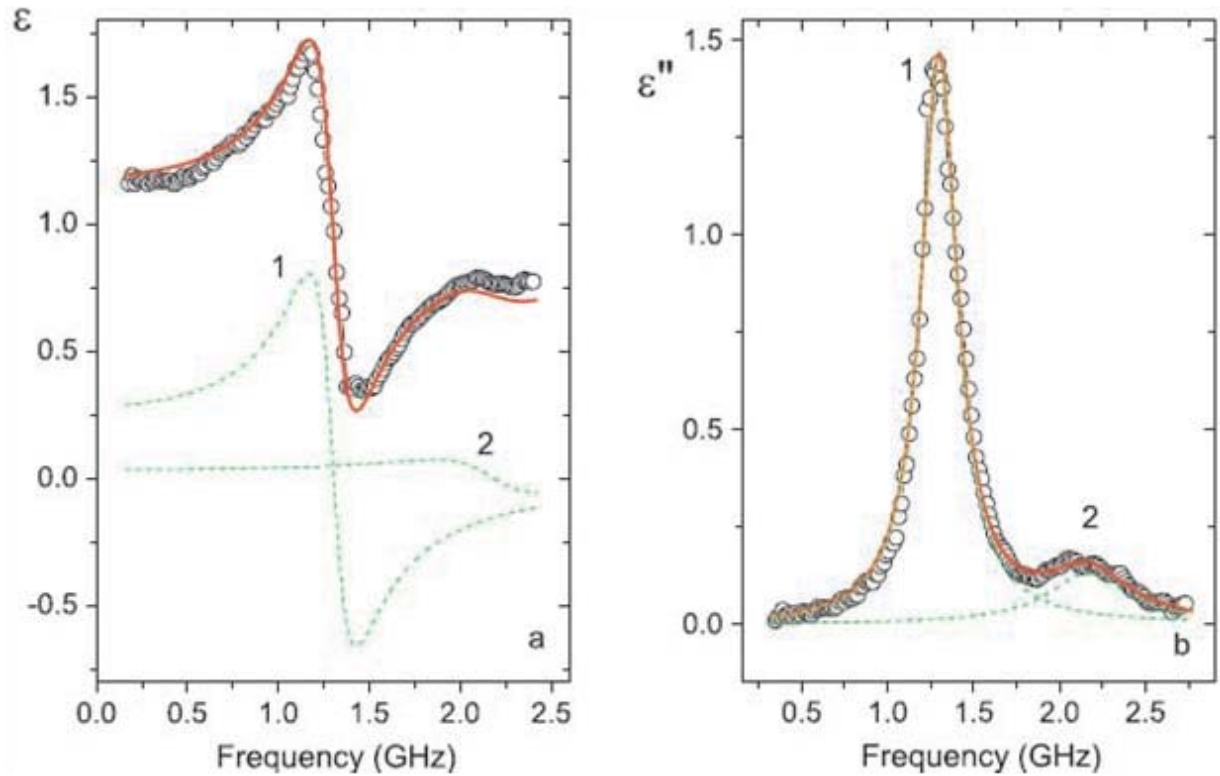

Fig. 8. The results of approximation of the real (a) and imaginary (b) parts of the dielectric permittivity for PMMA+TEACCB with NCs concentration of 3\% using equation (2). Peaks 1 and 2 are shown by the dotted lines. The solid line is the sum of fittings with $\varepsilon_{c}=0.871$.

\begin{tabular}{|c|c|c|c|}
\hline Peak & $\omega_{0} / 2 \pi(\mathrm{GHz})$ & $A /(2 \pi)^{2}\left(\mathrm{GHz}^{2}\right)$ & $\gamma / 2 \pi(\mathrm{GHz})$ \\
\hline \hline 1 & 1.305 & 0.487 & 0.129 \\
\hline 2 & 2.181 & 0.161 & 0.2876 \\
\hline
\end{tabular}

Table 1. Fitting parameters used for simulation of the resonance spectra of PMMA+TEACCB (3\%).

Using equation (2) we can approximate the experimental spectra (see Fig. 8). It was found that the high frequency tail of $\varepsilon^{\prime \prime}$ of TEACCB is similar to that for pure PMMA and, therefore, was subtracted. For the best fit it is necessary to take several resonance curves. Some discrepancy between the fitting curve and the experimental data of the real part of $\varepsilon$ in the high frequency region is connected with the contribution of the dielectric dispersion of the PMMA matrix which, in this case, could not be simply taken into account. Two different resonance curves may correspond to either thickness or radial extension mode. Although the designation of these modes concerns the macroscopic sample, their frequencies are determined by the corresponding sizes of the NCs. The 


\section{KAPUSTIANYK}

parameters of approximation are presented in Table 1. The considerable width of the resonance region is more likely connected with the dispersion of the sizes of the nanocrystals and their orientation in the sample.

On the other hand, it is generally accepted to treat the object of piezoelectric resonance around the resonant frequency as the electrical equivalent LCR circuit $[49,50]$. The resonant frequency $f r$ may be given as:

$$
f_{r} \cong \frac{1}{2 d} \sqrt{\frac{c_{\mathrm{eff}}}{\rho}},
$$

where $d$ is the average diameter of $\mathrm{NCs}$, and $c_{\text {eff }}$ and $\rho=1.45 \mathrm{~g} / \mathrm{cm}^{-3}$ are the effective elastic constant and the density of TEACCB NCs, respectively. As follows immediately from equation $(3), c_{\text {eff }}$ should be equal to $4.84 \cdot 10^{7}$ and $1.35 \cdot 10^{8} \mathrm{~Pa}$ in order to satisfy the experimentally observed values of resonant frequency $f_{r}=1.3 \mathrm{GHz}$ and $f_{r}=2.181 \mathrm{GHz}(d$ was put as $70 \mathrm{~nm})$. These values of $c_{\text {eff }}$ are two orders of magnitude smaller than those usually observed in other related ferroelectric bulk crystals (see, e.g., [51-54]). This discrepancy evidently is aparently connected with surface phenomena, such as a lateral (surface) tension characteristic of the nanostructured ferroics [55]. Therefore, the elastic properties should strongly depend on the size of NCs since the surface to volume ratio increases with the diminishing size of the NCs. The interface borders separating the nanocrystals and the polymer also modify considerably the elastic properties of the NCs [56]. The considered effects as well as the change of the poling electret field of the matrix cause a temperature shift of the resonance frequency.

\section{Conclusions}

Taking into account that no noticeable frequency dispersion was detected either for the TEACCB bulk crystal or for pure PMMA in the frequency region under investigation, the observed frequency dispersion of resonance type has to be treated as the physical phenomenon intrinsic to the composite material. The dielectric dispersion of the TEACCB NC-PMMA matrix compound reveals the resonance frequency dependence of both $\varepsilon^{\prime}$ and $\varepsilon^{\prime \prime}$ with resonant frequency near $1.3 \mathrm{GHz}$. The resonant frequency does not depend on the concentration of NCs in the host matrix, displaying at the same time the temperature shift to the lower frequencies upon cooling. The principal experimental findings presented in this part (occurrence of the resonance of dielectric permittivity, size independence of the resonance frequency and dependence of the resonance peak of $\varepsilon$ on $\mathrm{NC}$ concentration in the host matrix) may be explained as the result of the piezoelectric resonance of NCs. Since the bulk TEACCB crystal has the centric symmetry at room temperature, piezoelectricity in TEACCB NCs may only appear as a result of inverse symmetry violation within the electret PMMA matrix. The observed phenomenon could find its application in the creation of the principally new high frequency piezoelectric transducers.

\section{INFLUENCE OF SIZE EFFECTS ON THE PHASE TRANSITIONS AND THERMAL STABILITY OF THE NANOCOMPOSITES BASED ON FERROICS}

The single crystals of diethylammonium tetrachlorcuprtate (DEACC) cause substantial interest due to the high sensitivity of the $\mathrm{CuCl}_{4}$ complexes to the surrounding environmental background. It was established that the phase transitions observed at $311-330 \mathrm{~K}$ are dependent on the local environment of crystallites. We have observed substantial shift of the PT temperature for the $10 \mathrm{~nm}$ DEACC NC in the polymer PMMA matrices compared to the bulk materials. This is related to the manifestation of the nanosized effects in the investigated nanocomposites. The origin of the phenomenon is discussed within the framework of the existing nanoconfined models. Different regimes of cooling-heating cause different features of the phase transitions. For comparison we present the data of similar temperature measurements for the TEACCB nanocrystals incorporated into PMMA matrices.

\section{A. Experimental results and Discussion}

Following Fig. 9 and 10 one can see a substantial shift of the DSC maxima after the incorporation of the investigated NC into the PMMA matrix. The first peak of DSC for DEACC single crystals is formed by two peaks that would be related to the thermochromic phase transition and the temperature of crystal decay, respectively. Such a conclusion is confirmed by the fact that no DSC anomalies were observed at cooling after heating the sample above the decay temperature. Contrary to the case of single crystals the DSC anomaly for the nanocomposite is clearly separated onto two peaks, corresponding respectively to the phase transition and crystal decay. As follows from Fig. 9 the PT temperature is shifted from $47.2^{\circ} \mathrm{C}(320.2 \mathrm{~K})$ for the pure crystallites up to the $53.6^{\circ} \mathrm{C}(326.5 \mathrm{~K})$ for the composites. A giant temperature shift (from $305.1^{\circ} \mathrm{C}$ to $360.3^{\circ} \mathrm{C}$ ) of onset temperature for the TEACCB nanocrystals after their incorporation into the PMMA matrix was found. The observed shift is substantially larger compared to the DEACC. Similarly to the previous case the observed anomalies correspond to the crystal or composite decay. Such a conclusion is confirmed by the optical observations reflecting the lost of the optical quality of the material heated above the decay temperature. The analysis of the data presented in Fig. 10 testifies that in the case of the nanocomposite the DSC peak does not correspond to the decay of TEACCB NCs or the polymer matrix but hase to be related to the thermal decay of the entire composite system. Therefore, embedding the nanocrystals into the polymer matrix provides a growth of its thermal stability. 


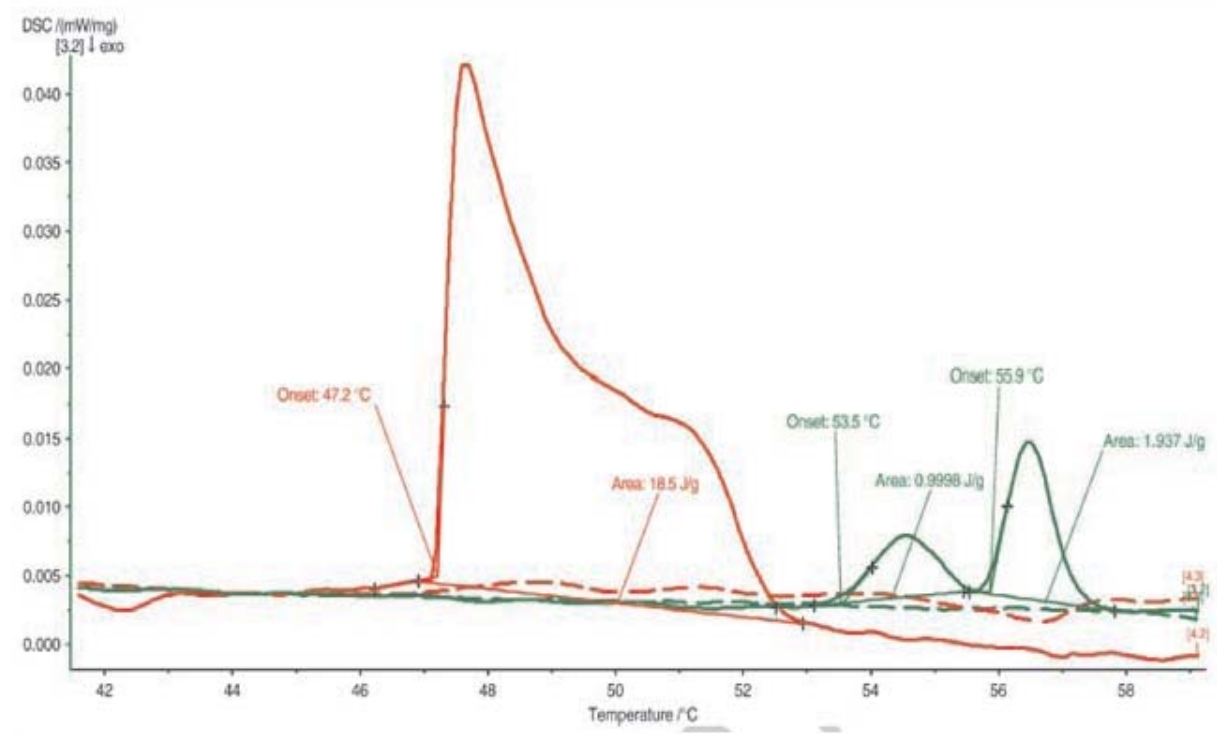

Fig. 9. Temperature dependence of the DSC for bulk (red line) and 20\% DEACC NCs incorporated into the PMMA matrices (green line). Temperature rate $0.3 \mathrm{~K} / \mathrm{min}$. (For interpretation of the references to color in this figure legend, the reader is referred to the web version of this article.)

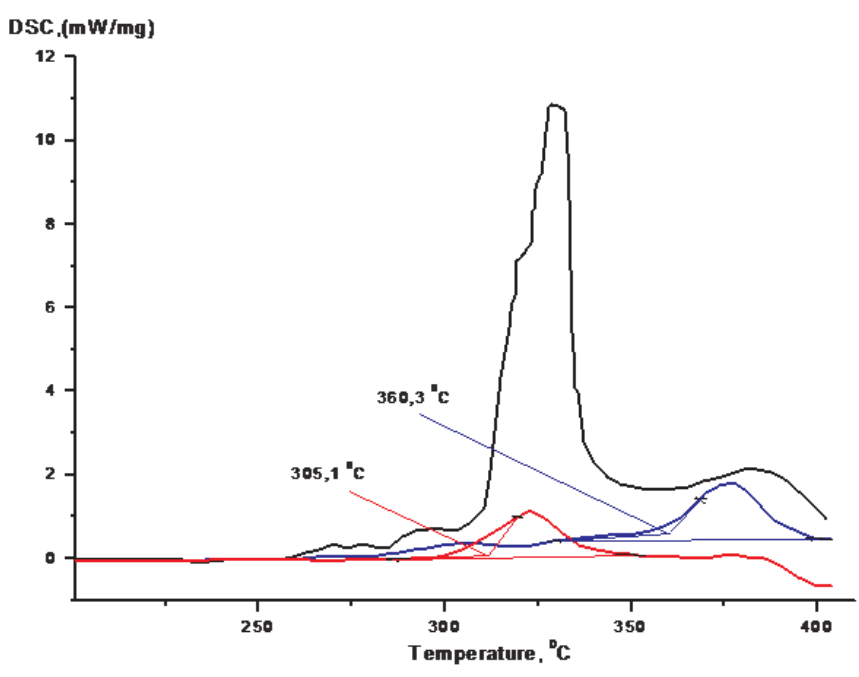

Fig. 10. (Color online) Temperature dependence of the DSC for bulk (red lower line), PMMA polymer (black upper line) and 5\% TEACCB NC incorporated into the PMMA matrices: (blue middle line) at temperature rate $10 \mathrm{~K} / \mathrm{min}$.

The obtained results unambiguously show that the principal role here is played by the interface borders separating the NCs and polymers. It is crucial to emphasize that due to the incorporation of the nanocrystals into the polymer matrices the surface effects will play an important role. First of all, this concerns arising of the surface tension and depolarising field [35]. The latter is closely connected with the electret state of the PMMA matrices discovered in Ref. [57]. Following the proposed model one can assume that the surrounding polymer PMMA matrix is highly polar. So the charge density changes on the borders NC-polymer are nonlinear with the temperature changes and we deal with the influence of local electro- static field shifting phase transition as well as thermal decay temperatures.

One can not exclude that the observed dependences may be also explained by intercrystallite interactions [58] and band energy flattering due to strong nanointerface electrostatic potential $[59,60]$.

\section{B. Conclusions}

A giant temperature shift (from $305.1^{\circ} \mathrm{C}$ to $360.3^{\circ} \mathrm{C}$ ) of the decay temperature both for TEACCB NC and polymer after the formation of the nanocomposite on their basis was found. The results unambiguously show that the principal role here is played by the interface borders separating the NC and polymers. The observed shift is substantially larger as compared to the DEACC composites. The obtained data testify to the fact that embedding of the nanocrystals into the polymer matrix provides a growth of their thermal stability. Such a conclusion is very important for the practical application both of the considered nanocomposites and polymers.

\section{MANIFESTATION OF THE SIZE EFFECT IN THE NONLINEAR OPTICS EFFECTS AND SPECTRAL PROPERTIES OF THE FERROIC NANOCRYSTALS INCORPORATED INTO THE POLYMER MATRIX}

In this part we would like to demonstrate that the incorporation of the nanocrystals into the polymer matrix drastically change their optical spectra and nonlinear optical properties. The investigations of the nonlinear optics effects in TEACCB NCs incorporated into olygoetheracrylate (OEA) matrix have shown that the in- 


\section{KAPUSTIANYK}

tensity of the photoinduced second harmonic generation decreases with the decreasing of crystallite sizes [8]. The choice of OEA as well as polymethylmethacrylate (PMMA) matrices allows to keep the nanocrystals in the relatively low strained surrounding background contrary to the traditional high stiffness polymers.

\section{A. Absorption spectra of the nanocomposites based on TEACCB}

The absorption spectra corresponding to the internal transition of the $\mathrm{Co}^{2+}$ ion for the TEACCB bulk and nanocrystals of different sizes incorporated into the OEA matrix clearly reflect the size effect (see Fig. 11). In order to compare the data for different samples the spectra corresponding to the nanocrystals were normalized with accounting of the cobalt ion concentration in a sample. As is clearly seen from Fig. 11, the absorption spectra of the NCs are characterized by the pronounced complex structure. Indeed, instead of one intensive slightly asymmetric broad band for the bulk material, for the case of the NCs one can observe the new bands arising at the high energy part of the spectra. Their energies are the higher the lower the crystallite size. Besides, one can observe a clear splitting of the main intensive band that becomes the most pronounced for the case of the smallest NCs.

In order to perform an unambiguous assignment of the observed absorption band the spectra have to be approximated with the Gaussian line-shapes (Fig. 12). The identification of the elementary bands was performed on the basis of the corresponding spectroscopic study of single crystals [13]. The A and B bands for the bulk material should be related to the spin allowed and spin forbidden electron transition on the degenerated energy levels, respectively [13] (see Table 2). In the case of the absorption spectra of the NCs the corresponding terms are split due to the lower symmetry of the crystal field. The degree of this splitting is the more pronounced the lower are the cluster sizes. On the basis of the obtained data one can conclude that such a splitting is connected with the distortion of the metal-halogen complexes within the surface layers of the nanocrystals due to the surface tension. One can expect that the ratio of these distorted surface complexes increases with the diminishing of the cluster sizes. It means that the most pronounced splitting of the two initial absorption bands has to be observed for the case of the smallest NCs that is really observed in the experiment. It is also necessary to note that the oscillator strength of the two initial bands observed in the bulk crystal is "distributed" between the larger number of bands in the samples with NCs that implies a noticeable decrease of absorption in the framework of $1.5-2.0 \mathrm{eV}$.

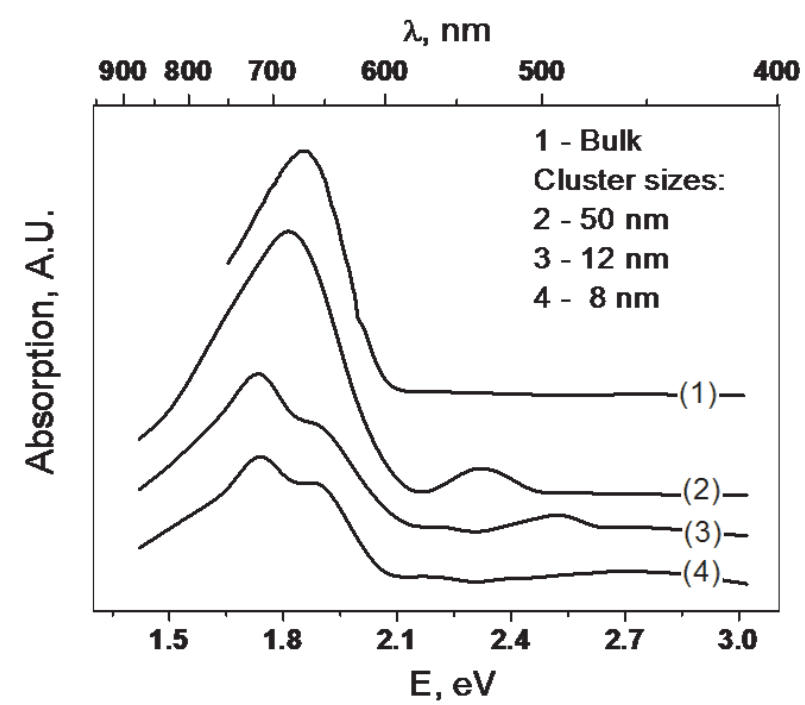

Fig. 11. Absorption spectra corresponding to the internal transition of $\mathrm{Co}^{2+}$ ion for the TEACCB bulk and nanocrystals of different sizes incorporated into olygoetheracrylate matrix.

\begin{tabular}{|c|c|c|c|c|c|}
\hline \multirow{2}{*}{$\begin{array}{c}\text { Transition from } \\
{ }^{4} \mathrm{~A}_{2}\left({ }^{4} \mathrm{~F}\right) \text { on }\end{array}$} & \multirow{2}{*}{ Bulk } & \multicolumn{4}{|c|}{ Nanocrystals } \\
\cline { 3 - 6 } & & $70 \mathrm{~nm}^{* *}$ & $50 \mathrm{~nm}$ & $12 \mathrm{~nm}$ & $8 \mathrm{~nm}$ \\
\cline { 2 - 6 } & & & 1.67 & 1.71 & 1.67 \\
\hline & 1.75 & - & 1.86 & 1.73 & 1.74 \\
\hline${ }^{4} \mathrm{~T}_{1}\left({ }^{4} \mathrm{P}\right)$ & & & & 1.92 & 1.90 \\
\hline & 1.90 & - & 2.33 & 2.49 & 2.71 \\
\hline${ }^{2} \mathrm{E}\left({ }^{2} \mathrm{G}\right)$ & & & & \\
\hline $\begin{array}{c}\text { Charge transfer band* } \\
X n p \rightarrow \text { Co3d }\end{array}$ & & 4.20 & - & - & - \\
\hline
\end{tabular}

(* Xnp: $\mathrm{Cl} 3 p$ or $\mathrm{Br} 4 p)$

(** incorporated into PMMA matrix)

Table 2. Parameters of the bands of the TEACCB bulk and NCs incorporated into OEA-matrix. 

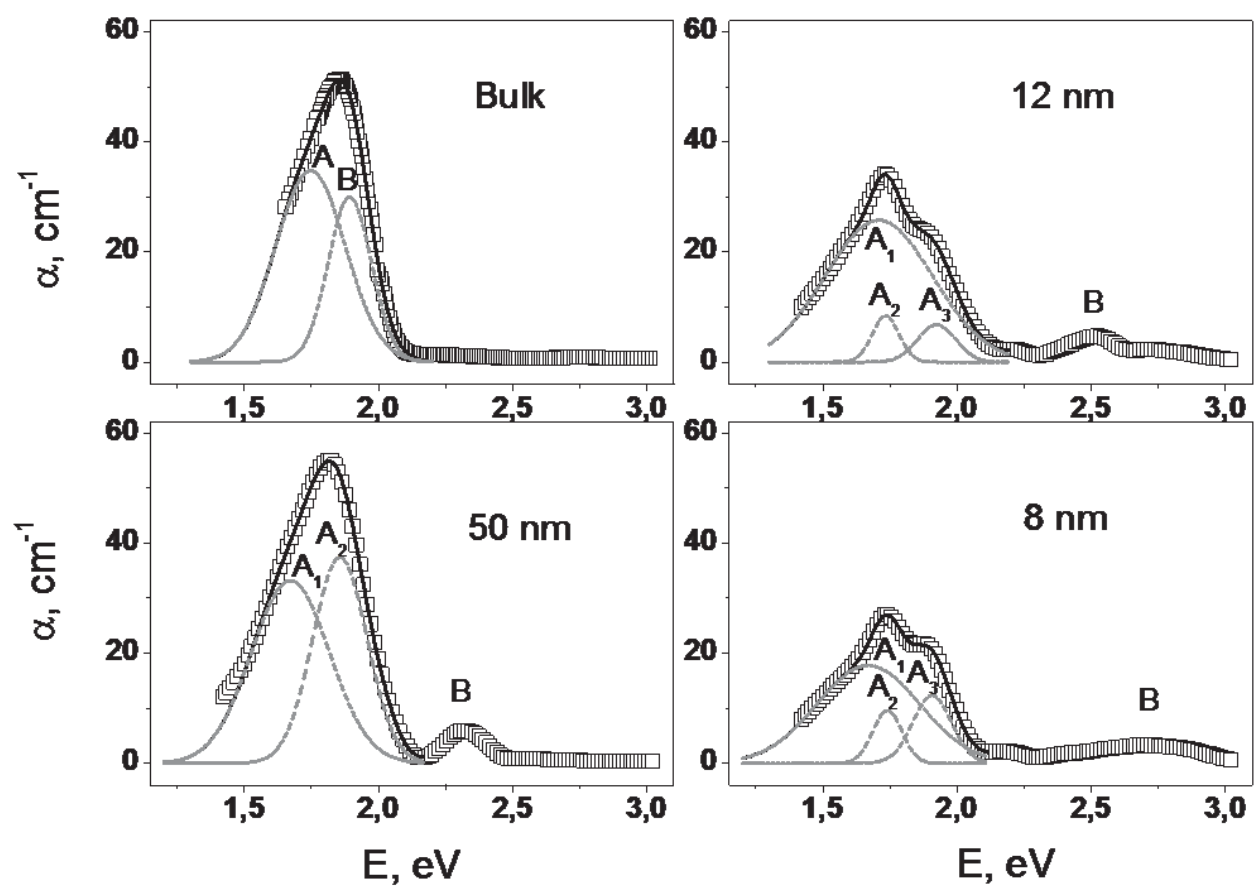

Fig. 12. Approximation of the absorption spectra corresponding to the internal transition of $\mathrm{Co}^{2+}$ ion by Gaussian lineshapes for bulk and NCs of the TEACCB incorporated into OEA-matrix.

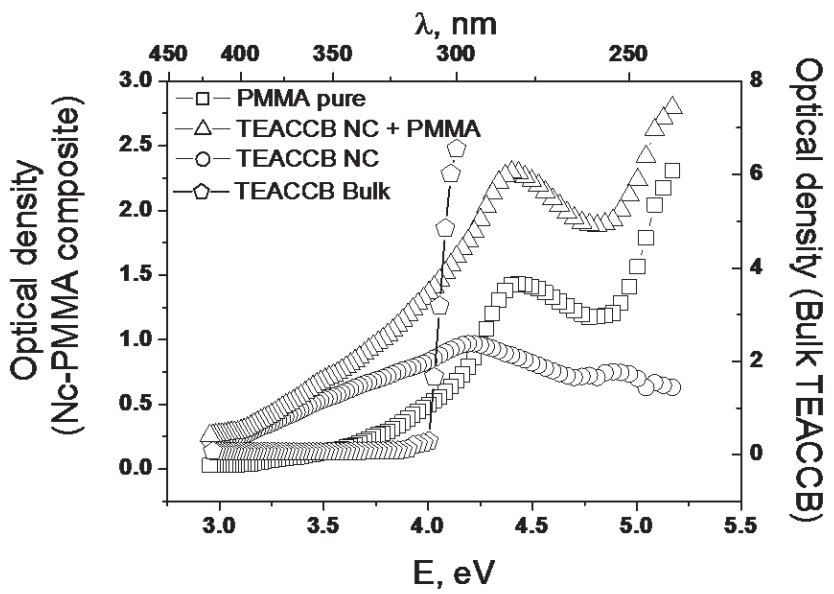

Fig. 13. The high energy part of the absorption spectra of the TEACCB: bulk (pentagons), pure PMMA (squares), composite (PMMA+TEACCB) (triangles), nanocrystals $(\sim 70 \mathrm{~nm})$ (circles).

This fact has to be taken into account in the nonlinear optics investigations since the wavelength of the light used for pumping also lies in the same region. At the same time, a clear blue shift of the high energy bands with the decreasing of the cluster size would be considered as manifestation of the quantum size effect. Indeed, the confinement of the electrons and holes within the narrow potential well implies an increase of their kinetic energy [61]. Taking into account that absorption in the high energy region of the spectra corresponding to the charge transfer transitions was found to be very high for the TEACCB NCs in the OEA-matrix, we have prepared samples with NCs incorporated into the polymethylmethacrylate matrix. This allowed us to identify clearly the complex charge transfer band in the vicinity of $4.20 \mathrm{eV}$ that cannot be observed in the bulk crystal due to a very large absorption in this spectral range (Fig. 13). In this case we take into account that the absorption edge of PMMA lies above $5 \mathrm{eV}$. Under such circumstances it is possible to obtain more detailed information concerning the entire energy diagram for TEACCB. Moreover, it is possible to estimate the energies of the charge transfer transitions not only for the NCs but also for the bulk crystals.

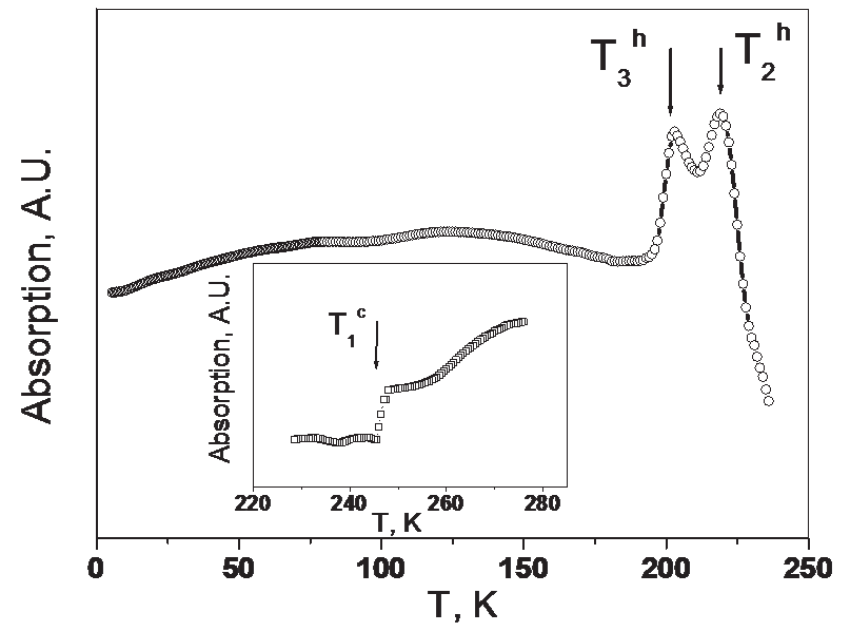

Fig. 14. Temperature dependences of absorption of the TEACCB nanocrystals incorporated into PMMA-matrix obtained at the constant wavelength $\lambda=350 \mathrm{~nm}$ in a heating and cooling (insert) runs.

The investigations of the considered composites containing the NCs offer a possibility to study the first or- 


\section{KAPUSTIANYK}

der phase transitions. They cannot be studied in a single crystal since the samples are cracked under the influence of internal tensions arising at the first order PT at $T_{2}$. The temperature dependences of absorption of TEACCB nanocrystals incorporated into the PMMA-matrix at constant wavelengths in the region of the charge transfer band measured in a wide temperature region are presented at Fig. 14. It is worth noting, that such dependences possess the global hysteresis in the temperature region of 160-320 K connected with the electret properties of PMMA matrix [44]. As it is clearly seen these dependences manifest anomalies at the temperatures of PTs close to those in a single crystal (see Table 3 ). However, below $T_{2}$ one can note an additional anomaly at $T_{3}$, which could be related to the previously unknown phase transition that could not be observed in a single crystal due to the above mentioned reason. It is worth noting that the PTs including those at $T_{3}$, were also observed in the temperature dependences of photoinduced second harmonic generation (PISHG) but at somewhat different temperatures. Under such circumstances one can conclude that the PT temperatures considerably depend on the type of polymer matrix used for preparing such composites (see Table 3).

\begin{tabular}{|c|c|c|c|}
\hline Sample & $T_{1}(\mathrm{~K})$ & $T_{2}(\mathrm{~K})$ & $T_{3}(\mathrm{~K})$ \\
\hline \hline TEACCB bulk & 249 & $224(232)$ & - \\
\hline $\begin{array}{c}\text { TEACCB NC (70 nm) in } \\
\text { PMMA-matrix }\end{array}$ & 247 & 219 & 202 \\
\hline (50 nm) in OEA-matrix & 238 & 225 & $\sim 210$ \\
\hline$(12 \mathrm{~nm})$ in OEA-matrix & 238 & 225 & $\sim 210$ \\
\hline$(8 \mathrm{~nm})$ in OEA-matrix & 237 & 230 & 223 \\
\hline
\end{tabular}

Table 3. The temperatures of the phase transitions in TEACCB - bulk and nanocrystals incorporated into a polymer matrix obtained at cooling (corresponding PT temperatures obtained for heating run are shown in parenthesis) on the basis of dielectric [8], optical spectroscopy and nonlinear optics data.

\section{B. Nonlinear optics effects in the nanocomposites based on TEACCB}

On the basis of the performed spectroscopic study one can make the conclusion concerning the nonlinear optics properties of the TEACCB nanocrystals incorporated into OEA-matrix. The temperature dependence of the photoinduced second harmonic generation demonstrates a remarkable dependence on temperature near the PTs (see Fig. 15). Indeed, for the samples with larger NCs the clear jumps are observed at the temperatures $T_{1}=238 \mathrm{~K}$ and $T_{2}=225 \mathrm{~K}$ (at cooling), close to the PT points in the bulk solid solution. The low temperature jump for the sample with the smallest nanocrystals is clearly shifted toward higher temperatures: $T_{2}=230 \mathrm{~K}$. One can also note the lower values of the PISHG in the latter case.
On the basis of the performed investigations one can conclude that the temperature of phase transition $T_{2}$ in the samples with the smallest NCs is higher than the one of the bulk material. The low temperature maxima on the corresponding dependences could be related to the phase transition at $T_{3}$. This $\mathrm{PT}$ is also shifted toward higher temperatures with the diminishing of the NC sizes. Taking into account the supposed improper ferroelectric origin of the phase lying in the framework of $T_{3}-T_{2}$ one can suggest that this effect is connected with the surface tension coupled with polarization (and order parameter) via electrostriction effect and the influence of the depolarization field arising around the surface of the nanocrystal [62]. As a result the improper ferroelectric phase is suppressed with the decreasing of the NC size. Such a size effect should be considered as a characteristic feature of the nanoferroelectrics [44].

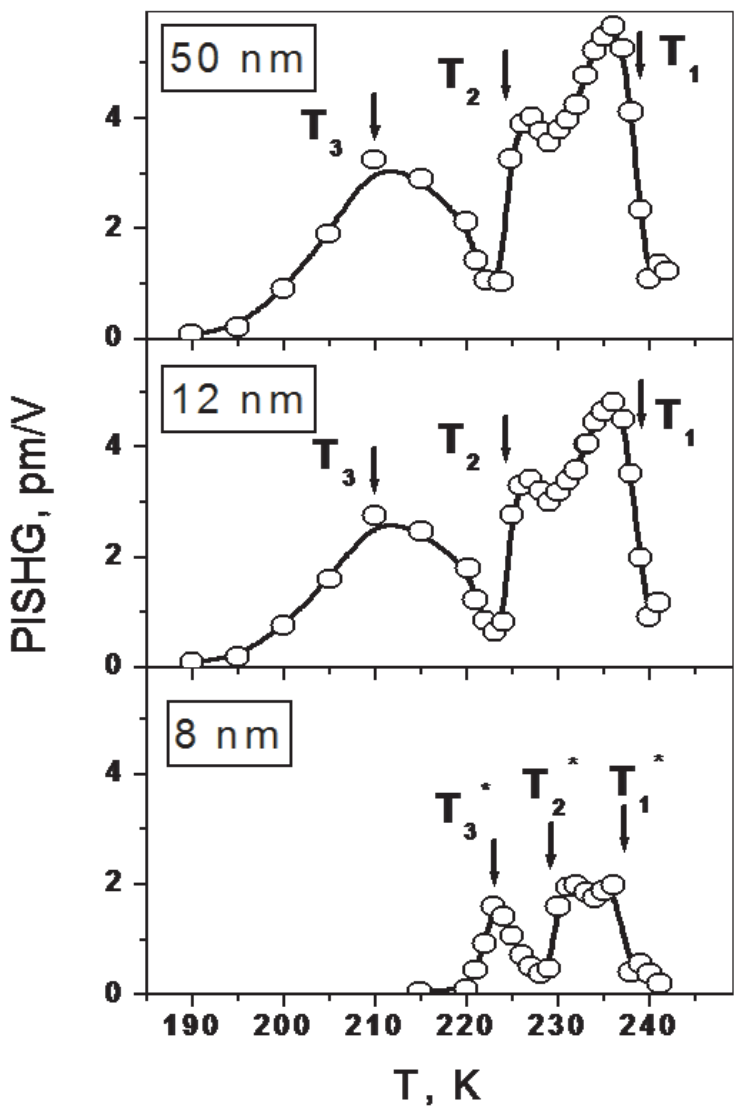

Fig. 15. Photoinduced SHG versus temperature for the TEACCB nanocrystals of different sizes incorporated into the OEA-matrix.

The shift of the corresponding absorption bands due to the lowering of the crystal field symmetry as well as the suppression of the intensity of the band A with diminishing of the NCs size is followed by considerable decreasing of PISHG intensity in all crystal phases.

The temperature $T_{1}$ of the high temperature phase transition for the TEACCB nanocrystals is also shifted in respect to the case of a bulk crystal. But this effect does not show clear size dependence and mostly is connected with the influence of the matrix type. 


\section{Influence of size effect on the thermochromic properties and charge transfer spectra of ferroics}

Technical application of the thermochromic DEACC crystals is restrained due to hygroscopicity and instability of their spectral parameters after repeated thermal recycling near the PT $[63,64]$. One can guess that incorporation of nanocrystals of mentioned compounds into the polymer matrix could resolve this problem. Besides, in such case one can observe the manifestation of the quantum confinement effects and related phenomena in the absorption spectra of such a type of materials. This part is devoted to detailed consideration of the size effects manifested in the spectral properties of the DEACC NCs embedded into the polymer matrices. The nanocomposites were prepared by two different methods [65]. In case of method 2 samples of certain size were selected using the membrane filter. The observed crystal field spectra were analyzed using the exchange charge model (ECM) of crystal fields.

The absorption spectra of the DEACC bulk crystals and the NCs in a visible and near infrared region are presented in Fig. 16 for comparison. The bulk crystals are characterized by asymmetric substantially broadened asymmetric bands in a low spectral energy part. They are originated from internal transition of $\mathrm{Cu}^{2+}$ ion. The corresponding electron transitions could be identified only using the approximation of the observed broad bands by Gaussian contours. Such a procedure is fairly described in Ref. [64]. The results of the band assignment are presented in Table 4 . In the high temperature "yellow" phase two bands corresponding to the electron transitions within the tetrahedral metal-halogen complexes are observed. Simultaneously, as follows from the obtained data, the low temperature "green" phase is characterized by coexistence of tetrahedral and octahedral coordinations of the copper ion. In the last case the crystalline field is much stronger that manifests itself in the clear shift of the corresponding bands (C, D and E) towards higher energies in comparison with the case of tetrahedral complexes. A sharp growth of absorption in the high energy spectral range of the bulk crystals should be related to the edge of the charge transfer (CT) bands. Unfortunately these bands cannot be entirely observed and identified due to a very large absorption.

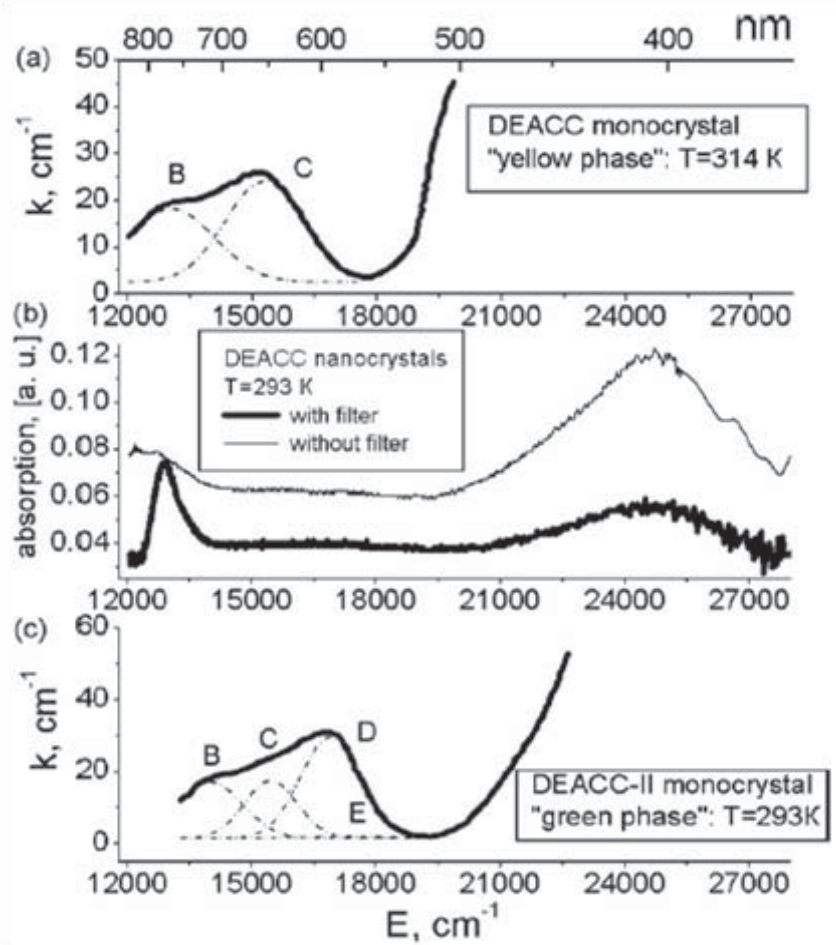

Fig. 16. Manifestation of the size effect in the absorption spectra of the DEACC crystals.

\begin{tabular}{|c|c|c|c|c|c|c|c|}
\hline \multirow[t]{4}{*}{ Band } & \multicolumn{5}{|c|}{ Transition energy, $\mathrm{eV}\left(\mathrm{cm}^{-1}\right)$} & \multicolumn{2}{|c|}{ Transition } \\
\hline & \multirow{2}{*}{\multicolumn{2}{|c|}{$\begin{array}{l}\text { Calculated energy } \\
\text { levels (ECM data) }\end{array}$}} & \multirow{2}{*}{\multicolumn{2}{|c|}{$\begin{array}{c}T(\mathrm{~K}) \\
\text { DECC bulk }\end{array}$}} & \multirow{3}{*}{$\begin{array}{c}\text { Nanocrystals } \\
(10 \mathrm{~nm}) \text { DEACC } \\
293 \mathrm{~K} \\
\end{array}$} & \multirow{3}{*}{$\begin{array}{r}\text { From } \\
d_{x^{2}-y^{2}} \\
\text { on } O_{h} \\
\end{array}$} & \multirow{3}{*}{$\begin{array}{c}\text { From } \\
d_{x y} \\
\text { on } T_{d} \\
\end{array}$} \\
\hline & & & & & & & \\
\hline & & & $293 \mathrm{~K}$ & $314 \mathrm{~K}$ & & & \\
\hline A & & & - & - & - & & $d_{y z x z}$ \\
\hline $\mathrm{B}$ & $\begin{array}{c}1.461 \\
(11778)\end{array}$ & $\begin{array}{c}1.564 \\
(12611)\end{array}$ & $\begin{array}{c}1.735 \\
(13980)\end{array}$ & $\begin{array}{c}1.623 \\
(13080)\end{array}$ & $\begin{array}{c}1.599 \\
(12890)\end{array}$ & & $d_{x^{2}-y^{2}}$ \\
\hline $\mathrm{C}$ & $\begin{array}{c}1.508 \\
(12161)\end{array}$ & $\begin{array}{c}1.871 \\
(15092)\end{array}$ & $\begin{array}{c}1.915 \\
(15430)\end{array}$ & $\begin{array}{c}.903 \\
(15325)\end{array}$ & - & $d_{z^{2}}$ & $d_{z^{2}}$ \\
\hline $\mathrm{D}$ & $\begin{array}{c}1.678 \\
(13522)\end{array}$ & $\begin{array}{c}2.183 \\
(17607)\end{array}$ & $\begin{array}{c}2.103 \\
(16935)\end{array}$ & - & - & $d_{x y}$ & \\
\hline $\mathrm{E}$ & $\begin{array}{c}2.038 \\
(16426)\end{array}$ & $\begin{array}{c}2.187 \\
(17 \quad 642)\end{array}$ & $\begin{array}{c}2.191 \\
(17655)\end{array}$ & - & - & $d_{y z, x z}$ & \\
\hline
\end{tabular}

Table 4. Identification of absorption bands corresponding to internal transition of $\mathrm{Cu}^{2+}$ ion for different phases of DEACC bulk crystal and for NC. 


\section{KAPUSTIANYK}

The spectra of nanocrystals (Fig. 16 b) considerably differs from the spectra of single crystals. First of all, for the NCs with the limited sizes dispersion $(<10 \mathrm{~nm})$, i.e. the samples obtained with using of filter, an appreciable narrowing of the absorption band corresponding to the band $\mathrm{B}$ of the bulk crystal (Fig. $16 \mathrm{~b}$ ) has been observed. The half-width of the band $\mathrm{B}$ is approximately eight times smaller than those for the corresponding bands for the bulk crystal. This phenomenon may be explained by the quantum confinement effect. Indeed, the number of interacting identical atoms confined in the nanocrystal is much smaller in comparison with that in the bulk crystal followed by a considerable narrowing of the $\mathrm{Cu}^{2+} 3 d$ energy levels in consequence of fulfillment of the Pauli principle. At the same time the half-width of the corresponding absorption band still remains finite due to the influence of the electron-phonon interaction. Another interesting factor is that in the high energy part one can observe entire CT bands since the absorption is not so high as in case of the bulk crystal. And finally, it is necessary to emphasize that a narrow single absorption band at $12890 \mathrm{~cm}^{-1}$ observed at room temperature may be related to the tetrahedral coordination of $\mathrm{Cu}^{2+}$ ion, whereas the bulk crystals are characterized by coexistence of two different complexes in the room temperature phase. This means that the octahedral complexes are suppressed in the NC and the thermochromic phase transition is not observed anymore. Indeed, the performed investigations show that no considerable changes were observed in the absorption spectra of the NCs near the temperature of thermochromic phase transition in the bulk crystals. On the basis of the obtained data one can conclude that such a phenomenon is caused by distortion of the metal-halogen complexes within the surface layers of nanocrystals due to the surface tension. One can expect that the ratio of these distorted surface complexes increases with diminishing cluster sizes. The arising of the lateral tension is a typical feature of the NCs of different types [66]. In our case this tension leads to the selection of the tetrahedral coordination from two possible shapes of the MHC.

To confirm validity of the identification of the crystal field spectra, we have used the ECM of crystal fields [67]. The main advantage of the ECM is that it allows to take into account the covalent effects for calculation of the values of the crystal field parameters from the crystal structure data. Since it operates with one fitting parameter only, it allows to achieve a good agreement between the calculated and experimental data for ions with unfilled electron shells in crystals. Taking into account that the detailed description of the ECM foundations and examples of their applications to various systems can be found in references [67-70] and references therein, we do not give here any further explanations, but provide the basic equation, which determines the form of the crystal field Hamiltonian. The energy levels of $\mathrm{Cu}^{2+}$ ion should be represented as the eigenvalues of the following Hamiltonian, which is defined as a linear combination of the irreducible tensor operators $O_{p}^{k}$ with crystal field parameters (CFP) as the coefficients of this combination [67]:

$$
H=\sum_{p=2,4} \sum_{k=-p}^{p} B_{p}^{k} O_{p}^{k}
$$

The equations for calculation of CFPs can be found in [67-70]. Taking into account that changes of MHC at the phase transition in the bulk crystals are described fairly well in the literature $[63,64,71-73]$, we consider in detail the case of tetrahedral coordination characteristic of the NCs. The $\mathrm{Cu}^{2+}$ ion in the tetrahedral $\left[\mathrm{CuCl}_{4}\right]^{2-}$ complex has only one term ${ }^{2} D$ which in the tetrahedral field $T_{d}$ is splitted upon two states: ${ }^{2} T_{2}$ (the ground state) and ${ }^{2} E$ (excited state). A further lowering of symmetry causes a further splitting of energy levels.

Analyzing the above mentioned energy levels for $\mathrm{Cu}^{2+}$ ion it is necessary to take into account that $3 d^{9}$ electron configuration has to be considered as the "hole analog" of the $3 d^{1}$ configuration. As a result, the sequence of levels for $3 d^{9}$ is inverse to those observed for $3 d^{1}$ (Table 4 ).

The spectral band around $12890 \mathrm{~cm}^{-1}$ in the NC corresponds to the electronic transition from the ground state to the lowest level of the ${ }^{2} E$ state. With this assignment, we have performed the ECM calculations of the crystal field parameters and $\mathrm{Cu}^{2+}$ energy levels. The calculated CFP values are shown in Table 5. Diagonalization of the Hamiltonian (4) with CFP from Table 5 in the space spanned by 5 wave functions of the ${ }^{2} D$ term (spin-orbit splitting was not considered, since the absorption bands are wide and no hyperfine structure was detected) resulted in the calculated energy level scheme shown in Table 4. The band A of the bulk DEACC for both coordination of the $\mathrm{Cu}^{2+}$ ions are fallen out of the photodetector sensitivity region.

\begin{tabular}{|c|c|c|}
\hline$B_{p}^{k}$ & $\begin{array}{c}\text { Tetrahedral } \\
\text { coordination }\end{array}$ & $\begin{array}{c}\text { Planar } \\
\text { coordination }\end{array}$ \\
\hline \hline$B_{2}^{-2}$ & 7426 & 90 \\
$B_{2}^{-1}$ & -1774 & 0 \\
$B_{2}^{0}$ & 4400 & -10052 \\
$B_{2}^{1}$ & 386 & 0 \\
$B_{2}^{2}$ & 7292 & 57 \\
$B_{4}^{-4}$ & -1681 & 98 \\
$B_{4}^{-3}$ & 427 & 0 \\
$B_{4}^{-2}$ & -7190 & -28 \\
$B_{4}^{-1}$ & -242 & 0 \\
$B_{4}^{0}$ & 2176 & 1419 \\
$B_{4}^{1}$ & -123 & 0 \\
$B_{4}^{2}$ & -6177 & -18 \\
$B_{4}^{3}$ & -957 & 0 \\
$B_{4}^{4}$ & 793 & 16552 \\
\hline
\end{tabular}

Table 5. CFP (in $\mathrm{cm}^{-1}$ ) for DEACC crystal. 
As is clearly seen from Table 4, calculated positions of the energy levels for the bulk crystal DEACC are in a reasonable agreement with the center of the absorption band B of DEACC NCs. The high energy part of the absorption spectra of the DEACC NCs is incorporated into the polymer matrix in different concentration are presented in Fig. 17. The clear CT bands were observed in the spectral range of $21000-26500 \mathrm{~cm}^{-1}$ for the samples prepared by the method 2 (Fig. $17 \mathrm{a}$ ). At the same time the corresponding CT bands for the samples prepared by the method 1 are much less resolved (Fig. $17 \mathrm{~b}$ ). This means that the technological treatment plays a substantial role in the formation of the high energy part of the absorption spectra. Indeed, employment of the filter in method 2 provides a more narrow dispersion of the NC sizes. Contrary to this feature a large dispersion of the NC sizes in samples obtained by method 2 causes a considerable broadening and overlapping of the CT absorption bands. It is also necessary to note the presence of the band in vicinity of $35000 \mathrm{~cm}^{-1}$ arising in result of superposition of the absorption bands intrinsic for the bulk DEACC crystal and PMMA matrix. The absorption edge of the polymers PMMA is observed in the high energy region of the spectra - in vicinity of $42000 \mathrm{~cm}^{-1}$ [74].

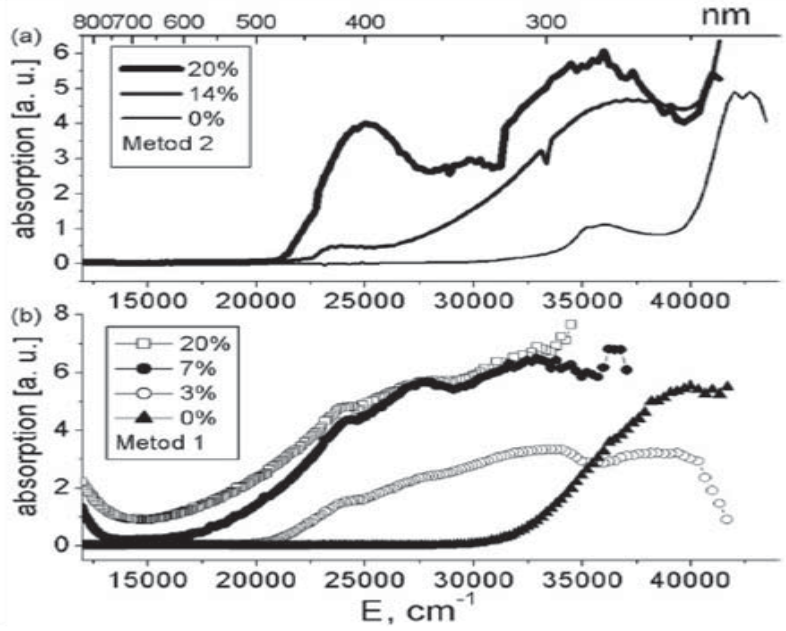

Fig. 17. The absorption spectra of DEACC nanocrystals incorporated into the PMMA matrix, recorded at room temperature.

In any case, following the obtained spectra of nanocomposites we have an opportunity to estimate an approximate energy of the charge transfer transitions that is impossible for the bulk crystals due to a very high absorption.

Let us try to identify the CT bands of ligand-metal types $\left(\mathrm{Cl} 3 p \rightarrow \mathrm{Cu} 3 d_{x^{2}-y^{2}}\right)$ in the spectral range of $21000-26500 \mathrm{~cm}^{-1}$. It is clear from Fig. 18 that there are three clear CT bands in this spectral range. It is necessary to consider the qualitative molecular orbital energy level diagram for $D_{2 d}$ symmetry of the $\mathrm{CuCl}_{4}^{2-}$ distorted by tetrahedral complexes [75]. In $T_{d}$ geometry, the 12 of C13 $p$ valence orbitals are divided into five molecular orbitals, that are split further in $D_{2 d}$ geometry into six $p(\pi)$ and three $p(\sigma)$ molecular orbitals. Between nine possible $\mathrm{CT}$ transitions, those from the $e$ molecular orbitals are $x, y$ polarized while those from the $a_{1}$ molecular orbitals are $z$ polarized [75]. Hence, five electric dipole allowed transitions are expected.

According to such a consideration we should observe in the unpolarized light the following absorption bands: band 1: ${ }^{2} A_{1} \leftarrow{ }^{2} B_{2}\left(3 a_{1}(\pi) 5 b_{2}\right)$; band 2: ${ }^{2} E$ $\leftarrow{ }^{2} B_{2}\left(4 e(\pi) 5 b_{2}\right)$; band $3:{ }^{2} E \leftarrow{ }^{2} B_{2}\left(3 e(\sigma) 5 b_{2}\right)$ (see Fig. 18).

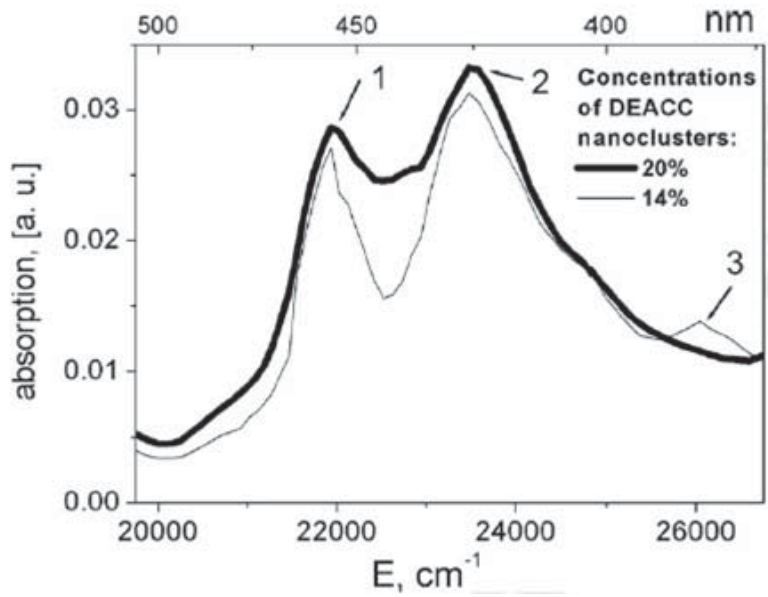

Fig. 18. Optical absorption spectra of DEACC nanocrystals (with average sizes $\simeq 10 \mathrm{~nm}$ ) of different concentrations in the region of the charge-transfer bands.

Table 6 presents values of the CT bands energy, calculated and obtained experimentally. The theoretically calculated oscillator strengths were taken from the work [76]. In these studies, only contributions from the ligand $3 p$ orbitals in the ground and excited states have been considered; this approximation has been demonstrated by van der Avoird and Ros to provide reasonable values for oscillator strengths. In this method, the wave function of a molecular orbital is written as

$$
\psi=C_{1} \Phi_{M}+C_{2} \chi_{L}
$$

where $\Phi_{M}$ is an atomic orbital of the central ion and

$$
\chi_{L}=\sum_{\alpha=1}^{4} \sum_{m_{l}=-1}^{1} C_{\alpha_{m l}} \Phi_{\alpha_{m l}}
$$

with $\Phi_{\alpha_{m l}}$ - atomic orbital of ligand $\alpha$ with the specified $m_{l}$ values, and $C_{1}$ and $C_{2}$ are the normalization constants. In the calculation of oscillator strengths, only integrals over orbitals on the same ligand are taken in to account, and the radial part of the $3 p$ wave function, which is a function of energy, is assumed to be invariant over a small energy range of transition. Thus the dipole moment integrals have the following form:

$$
\begin{aligned}
\left\langle\Phi^{\prime}|\mathbf{r}| \Phi\right\rangle & \approx C_{2}^{\prime} C_{2} \sum_{\alpha=1}^{4} \sum_{m_{l}=-1}^{1} C_{\alpha_{m l}}^{\prime} C_{\alpha_{m l}}\left\langle\Phi_{\alpha_{m l}}^{\prime}|\mathbf{r}| \Phi_{\alpha_{m l}}\right\rangle \\
& \approx C_{2}^{\prime} C_{2} \sum_{\alpha=1}^{4} \sum_{m_{l}=-1}^{1} C_{\alpha_{m l}}^{\prime} C_{\alpha_{m l}} \mathbf{r}_{\alpha}
\end{aligned}
$$




\section{KAPUSTIANYK}

where the primes indicate the excited state and $\mathbf{r}_{\alpha}$ is the position vector of ligand $\alpha$. Oscillator strengths are then calculated through the following formula

$$
f_{t h}=1.085 \cdot 10^{11} \bar{\nu}|D|^{2}
$$

where $\bar{\nu}$ is expressed in inverse centimeters and $D$ in centimeters.

Experimental oscillator strengths were determined through the approximation $f_{\exp } \approx 4.61 \cdot 10^{-19} \varepsilon_{\max } \bar{\nu}_{1 / 2}$, where $\bar{\nu}_{1 / 2}$ is the full width at a half maximum of the absorption band, $\varepsilon_{\max }$ - molar extinction coefficient [75]. The results of these calculations are presented in Table 6. The obtained $f_{\exp }$ values are only roughly approximate due to limited accuracy of evaluation of the DEACC NCs molar extinction coefficient as well as to contribution of the absorption of the matrix into oscillator strength of the absorption bands. Nevertheless the relative values of the oscillator strengths for allowed absorption bands observed experimentally are compatible with the calculated oscillator strengths for those allowed transitions.

\begin{tabular}{|c|c|c|c|c|c|c|}
\hline \multirow{2}{*}{$\begin{array}{l}\text { All possible CT } \\
\text { transitions }\end{array}$} & \multirow{2}{*}{$\begin{array}{l}\text { Calcul. trans. } \\
\text { energy, }\left(\mathrm{cm}^{-1}\right)\end{array}$} & \multirow{2}{*}{$\begin{array}{l}\text { Exper. obser. } \\
\text { trans. energy, } \\
\left(\mathrm{cm}^{-1}\right)\end{array}$} & \multicolumn{2}{|c|}{ Trans. $^{a}$ energy differences } & \multicolumn{2}{|c|}{ Oscillator strength ${ }^{b}$} \\
\hline & & & Calc. & Exper. & Theor. $^{b}$ & Exper. \\
\hline \multicolumn{7}{|l|}{$D_{2 d} \mathrm{CuCl}_{4}^{2-}$} \\
\hline${ }^{2} A_{2} \leftarrow{ }^{2} B_{2}\left(1 a_{2}(n b) \rightarrow 5 b_{2}\right)$ & 15440 & & & & & \\
\hline${ }^{2} E \leftarrow{ }^{2} B_{2}\left(5 e(\pi) \rightarrow 5 b_{2}\right)$ & 16360 & 21921 & 0 & 0 & 0.0349 & 0.0217 \\
\hline${ }^{2} A_{1} \leftarrow{ }^{2} B_{2}\left(3 a_{1}(\pi) \rightarrow 5 b_{2}\right)$ & 21620 & 23478 & 5260 & 1557 & 0.0004 & 0.00448 \\
\hline${ }^{2} B_{2} \leftarrow{ }^{2} B_{2}\left(4 b_{2}(\pi) \rightarrow 5 b_{2}\right)$ & 21790 & & & & & \\
\hline${ }^{2} E \leftarrow{ }^{2} B_{2}\left(4 e(\pi) \rightarrow 5 b_{2}\right)$ & 22680 & 24697 & 6320 & 2776 & 0.0721 & 0.00151 \\
\hline${ }^{2} B_{1} \leftarrow{ }^{2} B_{2}\left(1 b_{1}(\pi) \rightarrow 5 b_{2}\right)$ & 25040 & & & & & \\
\hline${ }^{2} E \leftarrow{ }^{2} B_{2}\left(3 e(\sigma) \rightarrow 5 b_{2}\right)$ & 26710 & 26119 & 10350 & 4198 & 0.0941 & 0.00163 \\
\hline${ }^{2} B_{2} \leftarrow{ }^{2} B_{2}\left(3 b_{2}(\sigma) \rightarrow 5 b_{2}\right)$ & 29430 & & & & & \\
\hline${ }^{2} A_{1} \leftarrow{ }^{2} B_{2}\left(2 a_{1}(\sigma) \rightarrow 5 b_{2}\right)$ & 33490 & & & & 0.199 & \\
\hline
\end{tabular}

${ }^{a}$ The transition energy differences are all tabulated with respect to the lowest energy charge-transfer transitions ${ }^{2} E \leftarrow{ }^{2} B_{2}\left(5 e(\pi) \rightarrow 5 b_{2}\right)$ in $D_{2 d}$

${ }^{b}$ For transitions from $e$ or $e_{u}$ orbitals, only the $x$ polarized component of the theoretical oscillator strength is given

Table 6. Correlations of the experimentally observed and calculated [76] energies of symmetry-allowed transitions and oscillator strengths for the $D_{2 d}$ symmetry of $\mathrm{CuCl}_{4}^{2-}$ complex in DEACC crystals.

\section{Size dependence of nonlinear properties of the nanocomposites based on DEACC}

Fig. 19 presents the size dependence of the third harmonic generation (THG) versus the NC sizes. One can clearly see that below $60 \mathrm{~nm}$ a drastic increase of the THG coefficients appears, achieving almost the same values at these low temperatures. And for the two-phonon absorption (TPA) (see Fig. 20) the corresponding values achieve $10 \mathrm{~cm} / \mathrm{GW}$ (LNT) and $8.8 \mathrm{~cm} / \mathrm{GW}(\mathrm{RT})$ for the diagonal TPA tensor components. For the off-diagonal values these tensor components are equal to $6.8 \mathrm{~cm} / \mathrm{GW}$ (RT), and $8.0 \mathrm{~cm} / \mathrm{GW}(\mathrm{LNT})$. At the same time, a typical behaviour of the TPA demonstrates a substantial increase with decreasing NC sizes (see Fig. 20), which may indicate a principal role of the nanoconfined states in the effects observed.

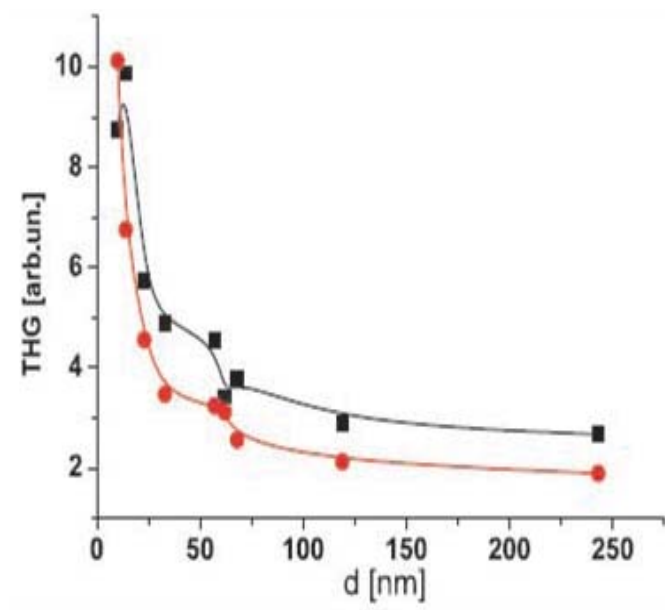

Fig. 19. Size dependence of the THG coefficients at RT (squares) and LNT (circles) for diagonal THG tensor components. 


\section{E. Conclusions}

On the basis of performed investigations it has been found that the temperatures $T_{2}$ and $T_{3}$ of phase transitions increase with decreasing of the TEACCB NCs size and are higher than those in the bulk material (at least for $T_{2}$ ). Taking into account the improper ferroelectric origin of the phase lying below $T_{2}$ one can suggest that this effect is connected with the surface tension coupled with polarization via electrostriction effect and the influence of the depolarization field arising around the surface of the nanocrystal. As a result the improper ferroelectric phase is suppressed. Besides, the arising lateral pressure induces lowering of the symmetry of the metal-halogen complexes within the surface layer of the cluster. Their distortion is followed by a more pronounced splitting of the degenerate energy levels due to the lower symmetry of the crystal field. At the same time the blue shift of the high energy elementary bands with decreasing of the cluster size is supposed to be due to the quantum confinement effect. The shift of the corresponding absorption bands as well as the suppression of the intensity of the integral absorption in the region of internal transition of $\mathrm{Co}^{2+}$ ion with diminishing of the NC size is followed by a considerable decreasing of PISHG intensity.

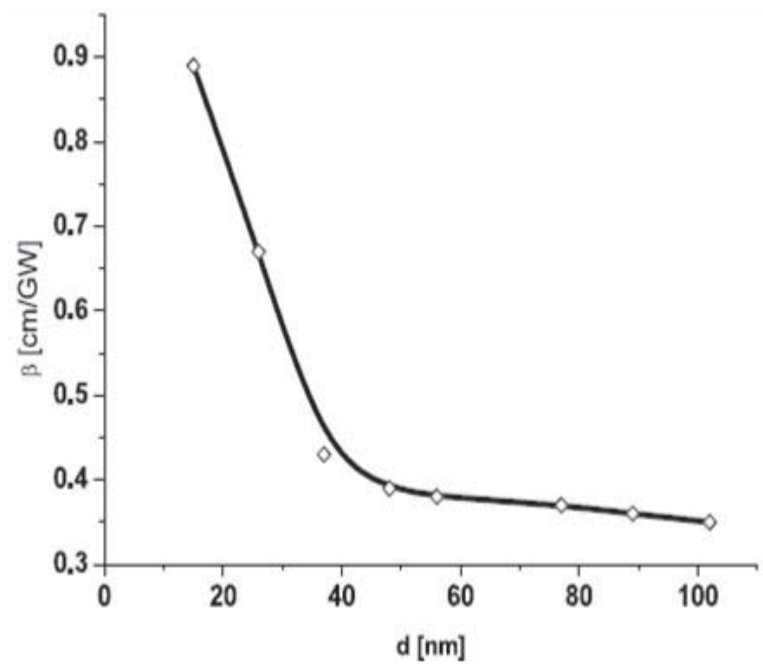

Fig. 20. Size dependence of the TPA for DEACC nanocrystals incorporated into the PMMA matrix, recorded at LNT for powder concentration about $20 \%$ at laser light power density $0.8 \mathrm{GW} / \mathrm{cm}^{2}$.

Experimental studies of the absorption spectra of the DEACC NCs embedded into PMMA showed that these spectra in the range of $12000-28000 \mathrm{~cm}^{-1}$ are considerably modified at transition from bulk crystals toward the nanocrystalline state. Such changes are caused by the specific symbiosis of the quantum confinement effects and specific surface phenomena. The quantum confinement effect in the NCs with sizes limited by $10 \mathrm{~nm}$ clearly manifests itself in a considerable narrowing of the absorption band corresponding to the internal transition of $\mathrm{Cu}^{2+}$ ion as a result of fulfillment of the Pauli principle. At the same time the half-width of this band still remains finite due to the influence of the electron-phonon interaction. It was shown that the lateral tensions arising on the surface of NC suppressed the octahedral coordination characteristic of the low temperature phase of the bulk crystal. As a result the thermochromic phase transition is not observed in the nanocrystals of the DEACC.

An incorporation of the NCs of DEACC into the polymer matrix allowed us to identify clearly the charge transfer bands of ligand-metal types $\mathrm{Cl} 3 p \rightarrow \mathrm{Cu} 3 d_{x^{2}-y^{2}}$ in the spectral range of $21000-26500 \mathrm{~cm}^{-1}$ that could not be observed in the absorption spectra of the bulk crystal. The theoretically calculated oscillator strengths for allowed CT bands agree fairly well with the oscillator strengths of the corresponding bands in the experimentally observed absorption spectra of the DEACC NCs. It was shown that the technological treatment of these nanocomposites, first of all an application of the membrane filters, plays a substantial role in the formation of the high energy part of the absorption spectra.

We observed a considerable enhancement of the thirdorder susceptibilities of the ferroic nanocomposite at the decrease of sizes of the DEACC NC size. For the NC sizes below $40 \mathrm{~nm}$ a drastic increase of the THG and TPA was found.

\section{SPECIFIC MAGNETIC AND DIELECTRIC PROPERTIES OF THE LOW DIMENSIONAL FERROICS}

The alkylammonium metal halides are twodimensional materials and exhibit strong structural anisotropy. They consist of inorganic layers of cornersharing $M X_{6}$ octahedra and organic layers of alkylammonium ions. Due to this peculiarity these materials undergoing a complicated sequence of PTs would be considered as the low dimensional ferroics. It has been found that due to the confinement of the excitons within the above mentioned layers of $n-\left(\mathrm{C}_{3} \mathrm{H}_{7} \mathrm{NH}_{3}\right)_{2} \mathrm{CdCl}_{4}$ crystals one can observe the enhancement of excitonic bands and specific manifestations of the radiation effects at quite high temperatures [77]. On the other hand it has been found that some of the two-dimensional alkylammonium copper halides $\left(\mathrm{C}_{n} \mathrm{H}_{2 n+1} \mathrm{NH}_{3}\right)_{2} \mathrm{CuCl}_{4}$ would be related to the multiferroics.

\section{A. Results and Discussion}

The temperature dependence of the magnetization for $\left(\mathrm{C}_{2} \mathrm{H}_{5} \mathrm{NH}_{3}\right)_{2} \mathrm{CuCl}_{4}$ (EACC) sample (Fig. 21) supports a picture where ferromagnetic-like interactions appear to play a significant role. The data for our EACC sample are in agreement with the pioneering work of de Jongh et al. $[21,78]$ who find a magnetic phase transition at $T_{c}=10.2 \mathrm{~K}$. The magnetization loops taken at different temperatures (Fig. 21, inset) show a quick saturation reaching $1 \mu_{B}$ per $\mathrm{Cu}^{2+}(S=1 / 2)$ at $5 \mathrm{~K}$, therefore supporting the presence of significant interactions below the magnetic ordering transition. It was claimed before that in a wide temperature range, the system shows 


\section{KAPUSTIANYK}

characteristics of a two dimensional Heisenberg ferromagnet with a dominant intralayer exchange coupling, $J / k_{\mathrm{B}}=18.6 \mathrm{~K}[78]$ for the spins at the nearest-neighbour $\mathrm{Cu}$ sites (Fig. 1). However, this behaviour is modified as one approaches $T_{c}$ from above because the interlayer coupling $J^{\prime}$ becomes significant. Despite the small magnitude of the latter $\left(J^{\prime} / J \approx-8 \cdot 10^{-4}\right)$ [79], the susceptibility resembles that of a system crossing over to a threedimensional ordered AFM array of FM layers (Fig. 1). This is a consequence of the superposition of intraplane FM interaction and a very weak AFM-hole-orbital ordering $\left(\ldots d_{x^{2}-z^{2}}-d_{y^{2}-z^{2}}-d_{x^{2}-z^{2}}-d_{y^{2}-z^{2}} \ldots\right)$ at the $\mathrm{Cu}^{2+}$ ions that occupy antiferrodistortively arranged $J-T$ elongated octahedra (within the $b c$ plane). This small antiferromagnetic coupling may be highly dependent on the interlayer distances and local lattice stresses that may additionally depend on materials' preparation procedure. Indeed the magnetic properties of this compound $(n=2)$ were suggested to be strongly pressure dependent [80] similarly to the pressure effects found in the case of the $n=1$ member of the family, i.e., $\left(\mathrm{CH}_{3} \mathrm{NH}_{3}\right)_{2} \mathrm{CuCl}_{4}$ [81]. Optical properties of EACC are also highly pressure dependent revealing a piezochromic effect [82].



Fig. 21. Magnetic field-cooled ( $H=10$ Oe) magnetization (left scale) and its inverse plot (right scale). Inset: magnetization as a function of magnetic field at different temperatures.

Although EACC has been known for a long time, the ferroelectric and dielectric properties have never been reported in the low-temperature region; likewise, an electric polarization has not been studied. It is however documented that EACC undergoes a complicated series of PTs, mostly connected with changes in the arrangement of the alkylammonium chains $[83,84]$. Bearing in mind the low temperature structural modifications here we report that the grown alkylammonium copper chloride crystals develop a large electric polarization when the material is cooled below about $247 \mathrm{~K}$. The temperature dependence of the real part of dielectric permittivity $\varepsilon^{\prime}$ obtained on cooling shows an anomaly in the region of 200-260 K, with a peak around $247 \mathrm{~K}$ confirming a phase transition (Fig. 22 a). It is worth noting that both the broad shape and the maximum value of $\varepsilon^{\prime}$ at $247 \mathrm{~K}$ are characteristic of improper ferroelectric PTs.
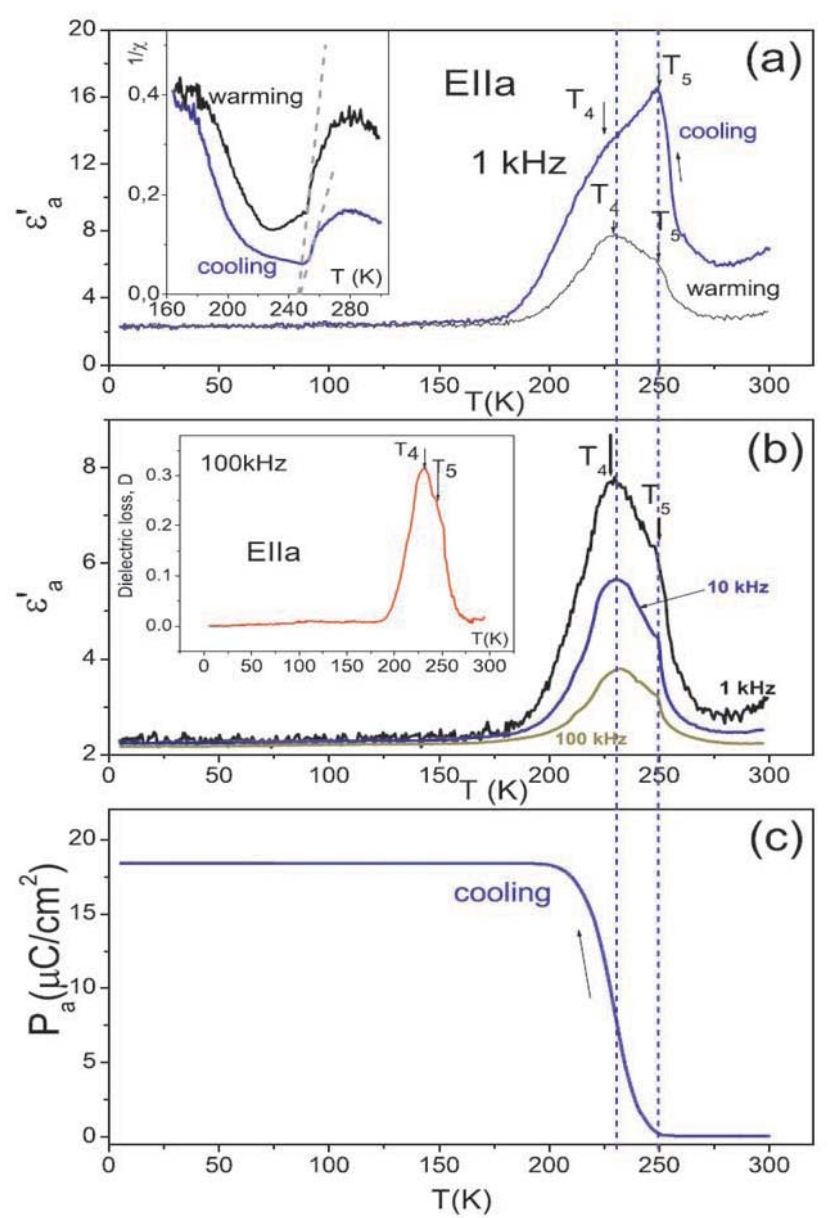

Fig. 22. The temperature evolution of the dielectric permittivity $\varepsilon^{\prime}$ for a EACC single crystal, (a) upon cooling and warming at $f=1 \mathrm{kHz}$; inset: the inverse dielectric susceptibility in the vicinity of the phase transition and (b) the corresponding dielectric dispersion at various frequencies (on warming); inset: the dielectric loss $D$, upon heating at $f=100 \mathrm{kHz}$. (c) shows the spontaneous electric polarization $P$ measured upon cooling.

To the first approach, one could suggest that anomalies in $\varepsilon^{\prime}$ on cooling are related to the same transition and the low temperature wing appearing below $T_{5}$ is related to the influence of domain-wall dynamics. However, in the corresponding data obtained upon warming, we can resolve two closely separated maxima at around $T_{4}=232 \mathrm{~K}$ and $T_{5}=247 \mathrm{~K}$ that are likely to arise from different types of structural ordering, characteristic for this compound $[83,84]$. An analysis of the inverse dielectric susceptibility $\chi^{-1}$ as a function of temperature (inset to Fig. 22 a) reveals a behaviour that follows the CurieWeiss law above $T_{5}$. Moreover, the linear extrapolation of $\chi^{-1}$ in the vicinity of the PT (temperature range of 250-255 K) intersects the $X$ axis at the same temperature, i.e., at $247 \mathrm{~K}$ both upon warming and cooling, thus pointing to a second-order type of PT at $T_{5}$. Under such 
circumstances the value $T_{5}=247 \mathrm{~K}$ should be considered as an exact phase transition temperature leading to polarization, while $T=232 \mathrm{~K}$ represents an additional transition temperature in the sample. Therefore, the obvious deviation from the Curie-Weiss behaviour (between $T_{5}$ and $T_{4}$, as well as below $T_{4}$ and the fact that maximum in $\varepsilon^{\prime}$ occurs at higher temperature on cooling $(247 \mathrm{~K})$ than on warming $(232 \mathrm{~K})$ are not features of the same transition (i.e., not a thermal hysteresis effect) but consequences of two closely situated phase transformations in the sample in the studied temperature range.

It is necessary to note that the temperature dependences of the dielectric permittivity at cooling and warming show different slopes in the range of $T_{4}$ and $300 \mathrm{~K}$. The main reason of such a behaviour can be due to ferroelastic domain structure [85]. The effect of hysteresis arises from the fact that we crossover the $T_{5}-T_{4}$ temperature range and come back to the room temperature so some ferroelastic domains [31] do not completely recover to their initial state. The temperatures at which the anomalies of the dielectric permittivity are observed $\left(T_{4}\right.$ and $T_{5}$ ) are independent of the electric field frequency $f$, confirming their relation to the PTs in EACC (Fig. 22 b). It is inferred that the anomalies in $\varepsilon^{\prime}$ should be the result of the order-disorder transitions connected with the reorientation of the alkylammonium groups as a whole. Organic chains flip among four equivalent orientations of the $\mathrm{NH}_{3}$ group inside the cavity of the $\mathrm{CuCl}_{6}$ octahdera which is a common feature in $\left(\mathrm{C}_{n} \mathrm{H}_{2 n+1} \mathrm{NH}_{3}\right)_{2} M_{\mathrm{Cl}}$ pervoskites $[27,28,86]$.

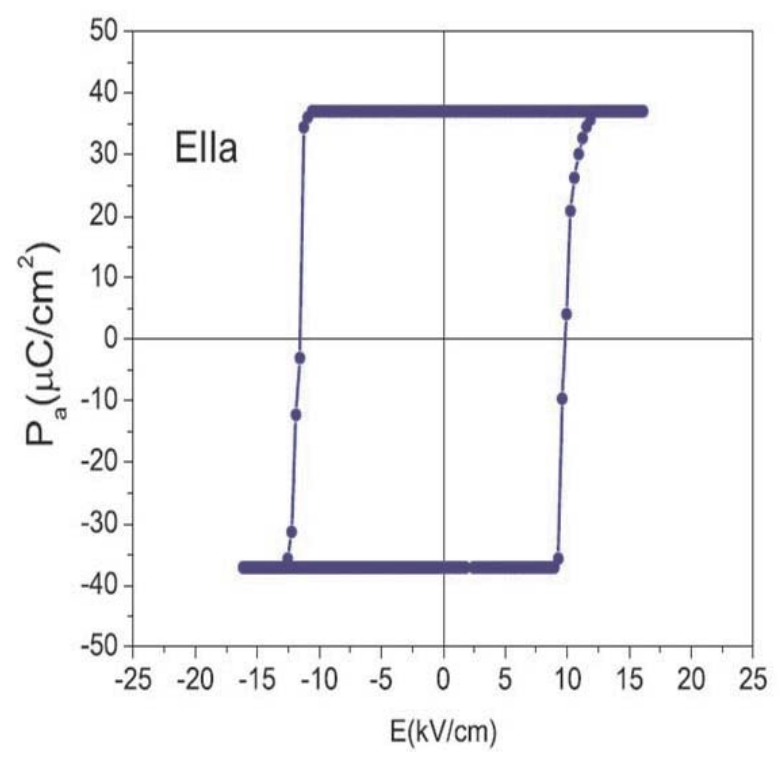

Fig. 23. Compensated ferroelectric hysteresis loop for a EACC single crystal at $77 \mathrm{~K}$.

The anomalies corresponding to the above-mentioned PTs were also seen in the temperature dependence of dielectric loss $D$ (inset Fig. $22 \mathrm{~b}$ ). The assumption concerning the occurrence of the intermediate phase between $T_{4}$ and $T_{5}$ correlates fairly well with investigations involving ferroelastic domains in this system [85]. According to the latter report there are two types of domains above $T_{5}$, one of which vanishes below this temperature $(247 \mathrm{~K})$, whereas below $T_{4}$ all domains disappear. Under such circumstances the intermediate phase would be considered as ferroelastic-improper ferroelectric one. In any case this suggestion needs further confirmation by an independent experimental method, such as X-ray diffraction or optical ferroelectric domains observation under polarized light.

In order to verify the polar response of the EACC we studied electric polarization by measuring an electric charge using automatic current integration with respect to time. Figure 22c shows the spontaneous electric polarization of the single-crystal sample measured upon cooling from $300 \mathrm{~K}$ to $5 \mathrm{~K}(3 \mathrm{~K} / \mathrm{min})$ without applied electric field. Indeed a large electric polarization, $P_{S}=18 \mu \mathrm{C} / \mathrm{cm}^{2}$, is found to develop at $T<T_{5}$. Moreover, by demonstrating below that this compound is also ferroelectric, we open the rare class of magnetic multiferroics to the rich family of organic-inorganic perovskites where distinct model magnetic and electrically polar compounds can be discovered. The ferroelectric behaviour is identified by the current integration with respect to the time method, which after leakage compensation has provided a well defined ferroelectric loop (Fig. 23) for EACC single crystal.

\section{B. Conclusions}

Therefore, we have provided compelling evidence that the $n=2$ member of the $\left(\mathrm{C}_{n} \mathrm{H}_{2 n+1} \mathrm{NH}_{3}\right)_{2} \mathrm{CuCl}_{4}$ family is a magnetic multiferroic low dimensional compound in which both electric polarization and dominant ferromagnetic interactions coexist. The EACC is a magnetic and ferroelectric compound that belongs to an emerging class of magnetic metal-organic multiferroics in which electric polarization is not induced by magnetic spin reorientation but ferroelectric and magnetic orders form independently. It is worth noting that a two dimensional character of the structure in this case is closely connected with co-existence of the ferro- and antiferromagnetic ordering that would be very important at further investigations of the magnetoelectric effects characteristic of the multferroics. Considering the abundance of compounds with general formula $\left(\mathrm{C}_{n} \mathrm{H}_{2 n+1} \mathrm{NH}_{3}\right)_{2} M X_{4}$, where $\mathrm{M}$ is a divalent metal, e.g., $\mathrm{Mn}^{2+}, \mathrm{Cd}^{2+}, \mathrm{Fe}^{2+}$ and $\mathrm{Cu}^{2+}$ and $\mathrm{X}$ is a halogen, the present results warrants screening other similar magnetic materials where two functional subsystems are in action. The highly tunable organic building blocks in conjunction with $3 d$ metal layers in hybrid perovskites offer an approach where electric order induced by hydrogen bonding can coexist with (ferro)magnetic phases and help to enrich the rare family of room-temperature multiferroics. In this regard one can note that not long ago we discovered the giant magnetodielectric effect in the new multiferroic compound $\left(\mathrm{N}\left(\mathrm{C}_{2} \mathrm{H}_{5}\right)_{4}\right)_{2} \mathrm{CoClBr}_{3}$ (TEACCB-3) [87], which by its chemical composition is very close to discussed above TEACCB. Now it is clear that TEACCB-3 also would be easily transformed into the nanocomposite 


\section{KAPUSTIANYK}

state using the same technology as for TEACCB. In such a way one can combine both nanoferroic and multiferroic in a one system.

\section{FINAL CONCLUSIONS}

The numerous experimental data presented in this review testify that transformation of the ferroics into the low dimensional state, first of all, due to formation of the nanocomposites drastically change their properties. Due to the specific symbiosis of the quantum confinement and other size effect one can observe principally new properties and effects:

1) dispersion of piezoelectric type in the ferroelectric nanocomposites (which were expected to be an isotropic material!) with a very high resonance frequency depending on the NC size;

2) clear shift of the phase transition temperatures at decreasing of the NC size;

3) suppression of the ferroelectric properties at decreasing of the NC size;

4) growth of thermal stability of the nanocomposites and corresponding polymer matrices due to embedding of the ferroelectric NCs;

5) suppression of the thermochromic properties due to the transformation from a bulk to NC state;

6 ) considerable narrowing and a blue shift of the crystal field bands at decreasing of the NC size;

7) considerable enhancement of the third-order susceptibilities of the ferroic nanocomposite at decrease of sizes of the nanocrystals;

8) co-existence of the ferro- and antiferromagnetic ordering in the two-dimensional ferroics that would be very important at further investigations of the magnetoelectric effects characteristic of the multferroics.

These principal experimental findings look even much more important when to take into account that due to embedding into a polymer matrix in the NC state the ferroic become very attractive from the technological point of view. The hygroscopic, brittle and thermally unstable crystals grown from aqueous solution are transformed by such a way that they would be used as a principally new nonlinear optics elements, piezotransducers, sensitive materials for sensors etc.

\section{ACKNOWLEDGEMENTS}

This review is based on the original papers published by the author in collaboration with: I. Kityk, K. Ozga (Technical University of Częstochowa, Poland), V. Rudyk, Ya. Shchur, M. Partyka (ScientificTechnical and Educational Center of Low Temperature Studies, I. Franko National University of Lviv, Ukraine), G. Lach, L. Laskowski, S. Tkaczyk, J. Swiątek, M. Piasecki, P. Brągiel (Jan Długosz University in Częstochowa, Poland), B. Kundys (Institute de Physique et de Chemie des Materiaux de Strasbourg, France), A. Lappas, I. Bakaimi (Institute of Electronic Structure and Laser, Foundation for Research and TechnologyHellas, Heraklion, Greece), M. Viret (Service de Physique de l'Etat Condese, DSM/IRAMIS/SPEC, CEA Saclay URA CNRS 2464, Gif-Sur-Yvette Cedex, France), Ch. Simon (Laboratoire CRISMAT, CNRS UMR 6508, ENSICAEN, Caen Cedex, France), M. Brik (Institute of Physics, University of Tartu, Estonia), K. J. Pluciński (Military University of Technology, Warsaw, Poland).
[1] M. D. Glinchuk, A. V. Ragulya, Nanoferroiki (Naukova dumka, Kiev, 2010) [In Russian]; M. D. Glinchuk, A. V. Ragulya, V. A. Stepanovich, Nanoferroics (Springer, 2013).

[2] P. E. Tomaszewski, Phase Transitions 39, 168 (1992).

[3] V. B. Kapustianyk, Fizyka krystalichnykh ferojikiv z orhanichnym kationom (Ivan Franko National University of Lviv, Lviv, 2006) [in Ukrainian].

[4] V. B. Kapustianik, Phys. Status Solidi B 204, 877 (1997).

[5] Z. Cummins, Phys. Rep. 185, 211 (1990).

[6] I. V. Kityk, R. I. Merwinskii, J. Kasperczyk, S. Jossi, Mater. Lett. 27, 233 (1996).

[7] F. Ramtolli, G. Saccinni, Cryst. Res. Technol. 37, 1325 (2002).

[8] V. Rudyk, I. Kityk, V. Kapustianyk, Nanocrystallites Ferroelectrics 330, 19 (2006).

[9] V. Kapustianyk, Ya. Shchur, I. Kityk, V. Rudyk, G. Lach, L. Laskowski, S. Tkaczyk, J. Swiątek, V. Davydov, J. Phys.: Cond. Matt. 20, 365215 (2008).

[10] A. J. Wolthuis, W. J. Huiskamp, L. J. De Jongh, R. L. Carlin, Physica B 142, 301 (1986).

[11] G. D. Stucky, J. B. Folkersand, T. J. Kistenmacher, Acta
Crystallogr. 23, 1064 (1967).

[12] P. Saint-Gregoire, H. Hunez-Bordallo, A. Mahoui, J. Lapasset, J. Moret, Ferroelectrics 190, 57 (1996).

[13] V. B. Kapustianyk, Y. M. Korchak, S. A. Sveleba, V. I. Mokryi, Zh. Prikl. Spektrosk. 61, 91 (2000).

[14] V. B. Kapustianyk, Zh. Prikl. Spektrosk. 67, 759 (2000).

[15] D. R. Bloomquist, M. R. Pressprich, R. D. Willett, J. Am. Chem. Soc. 110, 7391 (1988).

[16] A. Majchrowski, I. V. Kityk, J. Ebothe, Phys. Status Solidi B 241, 3047 (2004).

[17] M. Fiebig, J. Phys. D 38, R123 (2005).

[18] N. A. Spaldin, M. Fiebig, Science 309, 391 (2005).

[19] W. Eerenstein, N. D. Mathur, J. Scott, Nature (London) 442, 759 (2006).

[20] K. F. Wang, J.-M. Liu, Z. F. Ren, Adv. Phys. 58, 321 (2009).

[21] L. J. De Jongh, W. D. Van Amstel, A. R. Miedema, Physica 58, 277 (1972).

[22] M. Bibes, A. Barthelemy, Nature Mater. 7, 425 (2008).

[23] J. P. Steadman, R. D. Willett, Inorg. Chim. Acta 4, 367 (1970).

[24] K. Knorr, I. R. Jahn, G. Heger, Solid State Commun. 15, 231 (1974). 
[25] R. Kind, R. Blinc, B. Zeks, Phys. Rev. B 19, 3743 (1979).

[26] G. Chapuis, H. Arend, R. Kind, Phys. Status Solidi A 31, 449 (1975).

[27] R. Kind, Ferroelectrics 24, 81 (1980).

[28] G. Chapuis, R. Kind, H. Arend, Phys. Status Solidi A 36, 285 (1976).

[29] D. B. Mitzi, Prog. Inorg. Chem. 48, 1 (1999).

[30] I. R. Jahn, K. Holocher, K. Knorr, J. Ihringer, Z. Krystallogr. 174, 102 (1986).

[31] I. R. Jahn, K. Knorr, J. Ihringer, J. Phys.: Cond. Matt. 1, 6005 (1989).

[32] V. Kapustianyk, V. Rudyk, M. Partyka, Phys. Status Solidi B 244, 2151 (2007).

[33] L. G. Akselrud, Yu. N. Grin, P. Yu. Zavalii, V. K. Pecharsky, V. S. Fundamenskii, in 12th European Crystallographic Meeting (Collected Abstract) 3 (Nauka, Moscow, 1989), p. 155.

[34] G. Lach, L. Laskowski, I. Kityk, V. Kapustianyk, V. Rudyk, Ya. Shchur, S. Tkaczyk, J. Swiatek, M. Piasecki, J. Non-Cryst. Solids 353, 4353 (2007).

[35] M. Piasecki et al., Mater. Lett. 62, 2084 (2008).

[36] M. Piasecki et al., Chem. Phys. Lett.433, 136 (2006).

[37] J. Antonowicz, Wtasności dielektryków (Wydawnictwo Naukowo-Techniczne, Warszawa, 1971).

[38] A. K. Jonsher, Universal Relaxation Law (Chelsea Dielectric Press, London, 1996).

[39] P. C. Fannin, S. W. Charles, T. Relihan, J. Magn. Magn. Mater. 167, 274 (1997).

[40] G. D. Slucky, J. B. Folkers, T. J. Kistenmacher, Acta Crystallogr. 23, 1064 (1967).

[41] A. I. Wolthuis, W. J. Huiskamp, L. J. De Jongh, R. L. Karlin, Physica B 142, 301 (1986).

[42] K. Bolton, J. Lewis, O. Sprod, NEXUS: J. Undergrad. Sci. Eng. Technol. 1, 1 (2004).

[43] T. Kurosawa, J. Phys. Soc. Jpn. 16, 1298 (1961).

[44] K. Mazur, J. Phys. D: Appl. Phys. 30, 1383 (1997).

[45] C. S. Tu, F. T. Wang, R. R. Chien, V. H. Schmidt, L. C. Lim, J. Appl. Phys. 100, 074105 (2006).

[46] N. Cereceda et al., J. Eur. Ceram. Soc. 19, 1259 (1999).

[47] Yu. M. Poplavko, Fizika diélektrikov (Vyshcha Shkola, Kiev, 1980) [in Russian].

[48] V. Kapustianik et al., Ferroelectrics 202, 149 (1997).

[49] W. P. Mason, Phys. Rev. B 69, 173 (1945).

[50] Y. Xi, H. McKinstry, L. E. Cross, J. Am. Ceram. Soc. 66, 637 (1983).

[51] E. Nakamura, K. Kuramoto, J. Phys. Soc. Jpn 57, 2182 (1988).

[52] S. Prawer, T. F. Smith, T. R. Finlayson, Aust. J. Phys. 38, 63 (1985).

[53] P. Kubinec, E. Birks, W. Schranz, A. Fruith, Phys. Rev. B 49, 6515 (1994).

[54] A. V. Kityk, W. Schranz, A. Fuith, D. Havlik, V. P. Sopronyuk, H. Warhanek, Phys. Rev. B 53, 3055 (1996).

[55] V. Kapustianyk, V. Rudyk, M. Partyka, I. Kityk, S. Tkaczyk, J. Phys. C 79, 012005 (2007).

[56] M. Piasecki et al., Mater. Lett. 61, 1142 (2007).

[57] K. Mazur, J. Phys. D: Appl. Phys. 30, 1383 (1997) .

[58] B. Claudet et al., J. Opt. A: Pure Appl. Opt. 9, 315 (2007).

[59] I. V. Kityk, Semicond. Sci. Technol. 18 (12), 1001
(2003).

[60] I. V. Kityk et al., J. Phys.: Cond. Matt. 16, 231 (2004).

[61] S. Shionoya, W. M. Yen, Phosphor Handbook (CRC Press, Boca Raton, 1998).

[62] A. N. Morozovska, M. D. Glinchuk, E. A. Eliseev, Phase Transitions 80, 71 (2007).

[63] V. B. Kapustianik et al., Proc. 9th IEEE Int. Symp. Applications of Ferroelectrics, 801 (1994).

[64] V. B. Kapustyanyk, Yu. M. Korchak, J. Appl. Spectrosc. 67, 1045 (2000).

[65] V. Kapustianyk et al., J. Alloys Comp. 493, 26 (2010).

[66] A. N. Morozovska, M. D. Glinchuk, E. A. Eliseev, Phase Transitions 80, 71 (2007).

[67] Spectroscopy of Solids Containing Rare-Earth Ions, edited by B. Z. Malkin, A. A. Kaplyanskii, B. M. Macfarlane (North-Holland, Amsterdam, 1987).

[68] A. V. Savinkov et al., J. Phys.: Cond. Matt. 18, 6337 (2006).

[69] S. Bertaina et al., Phys. Rev. B 74, 184421 (2006).

[70] M. G. Brik, I. V. Kityk, Solid State Commun. 143, 326 (2007)

[71] V. P. Rudyk, I. V. Kityk, V. B. Kapustianyk, K. Ozga, Ferroelectrics 330,19 (2006).

[72] D. R. Bloomquist, R. D. Willett, H. W. Dodgen, J. Am. Chem. Soc. 103, 2610 (1981).

[73] S. A. Roberts, D. R. Bloomquist, R. D. Willett, H. W. Dodgen, J. Am. Chem. Soc. 103, 2603 (1981).

[74] D. R. Bloomquist, M. R. Pressprich, R. D. Willett, J. Am. Chem. Soc. 110, 7391 (1988).

[75] S. R. Desjardins, K. W. Penfield, S. L. Cohen, R. L. Musselman, E. I. Solomon, J. Am. Chem. Soc. 105, 4590 (1983).

[76] A. van der Avoird, P. Ros, Theor. Chim. Acta 4, 13 (1966).

[77] V. Kapustianyk, M. Panasyuk, M. Partyka, V. Rudyk, V. Tsybulskyy, Phys. Status Solidi B 246, 1686 (2009).

[78] B. Kundys, A. Maignan, D. Pelloquin, Ch. Simon, Solid State Sci. 11, 1035 (2009).

[79] L. J. de Jongh, H. Eugene Stanley, Phys. Rev. Lett. 36, 817 (1976).

[80] Y. Moritomo, Y. Tokura, J. Chem. Phys. 101, 1763 (1994).

[81] F. P. Missell, A. Paduan-Filho, Solid State Commun. 34, 527 (1980).

[82] K. Ohwada, K. Ishii, T. Inami, Y. Murakami, T. Shobu, H. Ohsumi, N. Ikeda, Y. Ohishi, Phys. Rev. B 72, 014123 (2005).

[83] V. Kapustianik, Yu. Korchak, S. Sveleba, R. Tchukvinskyi, I. Girnyk, Acta Phys. Pol. A 95, 351 (1999).

[84] M. J. Tello, J. L. Manes, J. Fernandez, M. A. Arriandiaga, J. M. Perez-Mato, J. Phys. C 14, 805 (1981).

[85] V. Kapustianik, Yu. Korchak, I. Polovinko, R. Tchukvinskyi, Z. Czapla, S. Dacko, Phys. Status Solidi B 20, 95 (1998).

[86] W. Depmeier, J. Felsche, G. Wildermut, J. Solid State Chem. 21, 57 (1977).

[87] V. Kapustianyk et al., in Abstracts of The 18 International Seminar on Physics and Chemistry of Solids (Lviv, Ukraine, September 12-15, 2012), p. 23. 


\title{
V. KAPUSTIANYK
}

\section{НАНОФЕРОЇКИ - НОВІ ЕФЕКТИ, ВЛАСТИВОСТІ, МОЖЛИВОСТІ}

\author{
В. Капустяник \\ Науково-технічний і навчальний центр низъкотемпературних досліджень, \\ Науково-навчальний центр "ФРАКТАЛ", кафедра фізики твердого тіла, \\ Львівсъкий університет імені Івана Франка, вул. Драгоманова, 50, 79005, Львів, Украӥна
}

Огляд присвячений аналізу найновіших результатів досліджень ефекту квантового запирання та інших розмірних ефектів у нанофероїках. Основну увагу приділено спектральним, діелектричним і нелінійнооптичним властивостям нанокомпозитів на основі монокристалів $\left(\mathrm{NH}_{2}\left(\mathrm{C}_{2} \mathrm{H}_{5}\right)_{2}\right)_{2} \mathrm{CuCl}_{4}$ (далі DEACC) i $\left(\mathrm{N}\left(\mathrm{C}_{2} \mathrm{H}_{5}\right)_{4}\right)_{2} \mathrm{CoCl}_{2} \mathrm{Br}_{2}$ (TEACCB). Розглянуті особливості магнітних і діелектричних властивостей на прикладі низькорозмірного фероїка $\left(\mathrm{C}_{2} \mathrm{H}_{5} \mathrm{NH}_{3}\right)_{2} \mathrm{CuCl}_{4}(\mathrm{EACC})$. Проаналізовано фізичні механізми принципово нових ефектів і властивостей наноструктурованих фероїків. Показано, що завдяки впровадженню в полімерну матрицю у вигляді нанокристалів фероїки стають дуже привабливими з технологічного погляду. Гігроскопічні, крихкі й термічно нетривкі кристали, вирощені з водних розчинів, трансформуються таким способом, що можуть бути застосовані як нелінійно-оптичні елементи, п'єзоперетворювачі, чутливі матеріали для сенсорів тощо. 